\title{
TWO-DIMENSIONAL SPACES IN WHICH THERE EXIST CONTIGUOUS POINTS*
}

\author{
BY \\ E. C. KLIPPLE
}

In a recent number of The Rice Institute Pamphlet $\uparrow \mathrm{R}$. L. Moore has formulated a set of axioms in terms of the undefined notions "point," "region," and "contiguous to." These axioms $\ddagger$ (Axioms A, B, C, 0, 1, and 2 of this paper) serve as the basis for the proofs of a considerable number of theorems of ordinary point set theory, including a large proportion of the theorems of the first two chapters of Moore's book. $\$$ Nevertheless, there exist spaces satisfying these axioms in which an arc may contain only a finite number of points and in which a region may consist of a finite number of points.

In the present paper a study is made of spaces which satisfy the above mentioned axioms and some additional axioms which restrict the spaces to being, in a certain sense, two dimensional. The ordinary euclidean plane is a space which satisfies all the axioms.

I wish to acknowledge my indebtedness to Professor R. L. Moore, and to thank him for suggesting the problem and for many helpful criticisms in the course of its development.

For definitions of terms used but not defined here the reader may refer to S.C.P.

Definitions. A simple closed curve is a compact continuum, containing at least two distinct non-contiguous points, which is disconnected by the omission of any two of its non-contiguous points. A triune is a set of three distinct points such that each of them is contiguous to each of the others.

Ахгом A. No point is contiguous to itself.

Axiom B. If the point $A$ is contiguous to the point $B$, then $B$ is contiguous to $A$.

* Presented to the Society, October 28, 1933; received by the editors August 18, 1937.

$\dagger$ Vol. 23 (1936), no. 1. In the present treatment the abbreviation S.C.P. will be used to designate part 1 of this paper.

It being understood that in the statement of Axiom 2 of S.C.P. the word "non-degenerate" is to be omitted. It is clear from the context that the retention of this word was not intended.

$\S$ Foundations of Point Set Theory, American Mathematical Society Colloquium Publications, vol. 13, New York, 1932. In the present treatment the abbreviation P.S.T. will be used to designate this book. 
Ахгом C. If $M$ is a closed point set and every point.of the set $H$ is contiguous to some point of $M$, then no point of $S-M$ is a limit point of $H$.

Aхгом 0. Every region is a point set.

Aхіом 1. There exists a sequence $G_{1}, G_{2}, G_{3}, \cdots$ such that (1) for each $n$, $G_{n}$ is a collection of regions covering $S$, (2) for each $n, G_{n+1}$ is a subcollection of $G_{n}$, (3) if $R$ is any region whatsoever, and if $X$ is a point of $R$ and $Y$ is a point of $R$ either identical with $X$ or not, then there exists a natural number $m$ such that if $g$ is any region belonging to the collection $G_{m}$ and containing $X$, then $\bar{g}$ is a subset of $(R-Y)+X$, (4) if $M_{1}, M_{2}, M_{3}, \cdots$ is a sequence of closed point sets such that $M_{n}$ contains $M_{n+1}$ for each $n$ and there exists a region $g_{n}$ of the collection $G_{n}$ such that $M_{n}$ is a subset of $\bar{g}_{n}$ for each $n$, then there is at least one point common to all the point sets of the sequence $M_{1}, M_{2}, M_{3}, \cdots$.

Aхгом 2. If $P$ is a point of a region $R$, there exists a connected domain containing $P$ and lying in $R$.

Aхгом 3. If $J$ is a simple closed curve or triune, then $S-J$ is the sum of two mutually separated connected point sets such that $J$ is the boundary of each of them.

In this paper I deal very frequently with complementary domains of simple closed curves and triunes. If $J$ is a simple closed curve or triune and $\omega$ is a point of $S-J$, then by the interior of $J$ with respect to $\omega$, is meant that complementary domain of $J$ which does not contain $\omega$. Similarly, that complementary domain of $J$ which contains $\omega$ is called the exterior of $J$ with respect to $\omega$. In case no ambiguity arises, the terms interior and exterior of $J$ will be used without making specific reference to a point $\omega$.

THeORem 1. Let $J_{1}$ and $J_{2}$ denote two point sets each of which is either a simple closed curve or a triune. Let $I_{1}$ and $I_{2}$ denote the interiors of $J_{1}$ and $J_{2}$, respectively, and suppose $J_{2}$ is a subset of $J_{1}+I_{1}$. Then $I_{2}$ is a subset of $I_{1}$.

THeorem 2. If $J$ is a simple closed curve, and $A B$ separate $C$ and $D$ on $J$, and $A X B$ is an arc such that the segment $A X B$ is a subset of $I$, the interior of $J$, and no point of the segment $A X B$ is constiguous to any point of $J-(A+B)$, then (1) $I_{1}$, the interior of $A X B C A$, is a subset of $I$, (2) the segment $A D B$ is a subset of the exterior of $A X B C A$, and (3) $I_{1}$ has no point in common with $I_{2}$, the interior of $A X B D A$.

Theorem 3. Under the hypothesis of Theorem 2, $I=I_{1}+I_{2}+$ segment $A X B$.

Proof. Suppose $I\left(I_{1}+I_{2}+\right.$ segment $\left.A X B\right)=M$, where $M$ is a non-vacuous point set. Let $P$ be a point of $M$. By Theorem 38 of S.C.P. there exists an $\operatorname{arc} P X$ from $P$ to $X$ lying in $I$. The $\operatorname{arc} P X$ contains an $\operatorname{arc} P X^{\prime}$ such that 
$P X^{\prime}-X^{\prime}$ is a subset of $M$, and $X^{\prime}$ is a point of the segment $A X B$. Similarly, there exists an arc $P \omega$ lying in $E_{2}$, the exterior of $A X B D A$. The arc $P \omega$ contains an arc $\dot{P} C^{\prime}$, such that $P C^{\prime}-C^{\prime}$ is a subset of $M$, and $C^{\prime}$ is a point of the segment $A C B$. Let $T$ denote the first point in the order from $X^{\prime}$ to $P$ which $X^{\prime} P$ has in common with $P C^{\prime}$. If no point of the interval $X^{\prime} T$ of $X^{\prime} P$, except possibly $T$, is contiguous to any point of $\left(T C^{\prime}-T\right)$, where $T C^{\prime}$ denotes the interval of $P C^{\prime}$ from $T$ to $C^{\prime}$, then $X^{\prime} T+T C^{\prime}$ is an arc from $X^{\prime}$ to $C^{\prime}$. If there exist points of $X^{\prime} T-T$ which are contiguous to points of $T C^{\prime}-T$, there must be a first such point in the order from $X^{\prime}$ to $T$. For otherwise, there would be infinitely many such points, and the set of all such points would have a limit point in $X^{\prime} T-T$. But by Axiom $\mathrm{C}$, every limit point of such a set must belong to $T C^{\prime}$, and a contradiction is reached. Let $W$ denote the first point of $X^{\prime} T-T$ which is contiguous to a point of $T C^{\prime}-T$. By Axiom $\mathrm{C}, W$ is contiguous to only a finite number of points of $T C^{\prime}-T$. Let $V$ denote the last point in the order from $T$ to $C^{\prime}$ which is contiguous to $W$. Let $X^{\prime} W$ denote the interval of $X^{\prime} T$ from $X^{\prime}$ to $W$ or the point $X^{\prime}$ according as $W$ is not or is identical with $X^{\prime}$. Let $V C^{\prime}$ denote the interval of $T C^{\prime}$ from $V$ to $C^{\prime}$ or the point $C^{\prime}$ according as $V$ is not or is identical with $C^{\prime}$. The point set $X^{\prime} W+V C^{\prime}$ is an arc containing at least three points. Thus, in any case, the point set $P X^{\prime}+P C^{\prime}$ contains an arc $X^{\prime} P^{\prime} C^{\prime}$, such that the segment $X^{\prime} P^{\prime} C^{\prime}$ contains at least one point and is a subset of $M$. Similarly, we may show the existence of an arc $X^{\prime \prime} P^{\prime \prime} C^{\prime \prime}$ such that (1) the segment $X^{\prime \prime} P^{\prime \prime} C^{\prime \prime}$ contains at least one point and is a subset of $I_{1}$ and (2) the points $X^{\prime \prime}$ and $C^{\prime \prime}$ are points of the segments $A X B$ and $A C B$, respectively. Let $X^{\prime} X^{\prime \prime}$ denote the point $X^{\prime}$ or the arc of the segment $A X B$ from $X^{\prime}$ to $X^{\prime \prime}$, according as $X^{\prime \prime}$ is or is not identical with $X^{\prime}$. Also let $C^{\prime} C^{\prime \prime}$ denote the point $C^{\prime}$ or the arc of the segment $A C B$ from $C^{\prime}$ to $C^{\prime \prime}$, according as $C^{\prime \prime}$ is or is not identical with $C^{\prime}$. By means of repeated applications of Axiom $C$ it may be shown that the point set $X^{\prime} P^{\prime} C^{\prime}+C^{\prime} C^{\prime \prime}+X^{\prime \prime} P^{\prime \prime} C^{\prime \prime}+X^{\prime} X^{\prime \prime}$ contains a simple closed curve $J^{\prime}$, which contains at least one point of each of the segments $X^{\prime} P^{\prime} C^{\prime}$ and $X^{\prime \prime} P^{\prime \prime} C^{\prime \prime}$. Let $I^{\prime}$ denote the interior of $J^{\prime}$. Now $I^{\prime}$ is a subset of $I$ by Theorem 1 . Thus the segment $A D B$ is a subset of $E^{\prime}$, the exterior of $J^{\prime}$. Since the connected set $I_{2}$ plus the segment $A D B$ contains no point of $J^{\prime}$ but does contain a point of $E^{\prime}$, it follows that $I_{2}$ plus the segment $A D B$ is a subset of $E^{\prime}$. Furthermore $I^{\prime}$ cannot contain a point of the segment $A X B$. For, suppose $I^{\prime}$ contains the point $F$ of the segment $A X B$. The connected set $F+I_{2}$ contains no point of $J^{\prime}$ but contains points of both complementary domains of $J^{\prime}$. We conclude from this contradiction that the segment $A X B$ is a subset of $J^{\prime}+E^{\prime}$. Now $I^{\prime}$ cannot be a subset of $M$ since there exists a point of $J^{\prime} \cdot I_{1}$ which is either a limit point of $I^{\prime}$ or contiguous to a point of $I^{\prime}$, 
and $M$ and $I_{1}$ are two mutually separated domains. Also, $I^{\prime}$ cannot be a subset of $I_{1}$ since there exists a point of $J^{\prime} \cdot M$ which is either a limit point of $I^{\prime}$ or contiguous to a point of $I^{\prime}$, and $M$ and $I_{1}$ are two mutually separated domains. Also $I^{\prime}$ cannot be a subset of $M+I_{1}$ and contain points of both $M$ and $I_{1}$ since $I^{\prime}$ would thus be the sum of two mutually separated sets, contrary to Axiom 3. Thus in any case we reached a contradiction, and the theorem is established.

TheOREm 4. If the points $A$ and $B$ separate the points $C$ and $D$ on the simple closed curve $J$, and if the segments $A X B$ and $C Y D$ are both subsets of $I$, the interior of $J$, then these segments have at least one point in common.

Proof. Suppose the segments $A X B$ and $C Y D$ have no point in common. There exists an $\operatorname{arc} A^{\prime} \omega$ such that $A^{\prime} \omega-A^{\prime}$ is a subset of $E$, the exterior of $J$, and such that $A^{\prime}$ is a point of the segment $C A D$ of $J$. Similarly, there exists an $\operatorname{arc} B^{\prime} \omega$ such that $B^{\prime} \omega-B^{\prime}$ is a subset of $E$ and $B^{\prime}$ is a point of the segment $C B D$. The point set $A^{\prime} \omega+B^{\prime} \omega$ contains an arc $A^{\prime} X^{\prime} B^{\prime}$ such that the segment $A^{\prime} X^{\prime} B^{\prime}$ is a subset of $E$. Let $A A^{\prime}$ denote the point $A$ or the arc of the segment $C A D$ from $A$ to $A^{\prime}$ according as $A^{\prime}$ is or is not $A$, and let $B B^{\prime}$ denote the point $B$ or the arc of the segment $C B D$ from $B$ to $B^{\prime}$ according as $B^{\prime}$ is or is not $B$. It may be shown that there exists a simple closed curve $J^{*}$ satisfying the following conditions: (1) $J^{*}$ is a subset of $A A^{\prime}+B B^{\prime}+A X B+A^{\prime} X^{\prime} B^{\prime}$, (2) $J^{*}$ contains at least one point of each of the segments $A X B$ and $A^{\prime} X^{\prime} B^{\prime}$, and (3) $J^{*} \cdot J$ is the sum of two mutually separated continua which separate $C$ and $D$ on $J$; therefore $J-J^{*} \cdot J=g_{1}+g_{2}$, where $g_{1}$ and $g_{2}$ are mutually separated segments containing $C$ and $D$, respectively. Since $J^{*}$ contains no point of the arc $C Y D$, it follows that the connected point set $C Y D+g_{1}+g_{2}$ is a subset of $I^{*}$, a complementary domain of $J^{*}$. Thus $J$ is a subset of $I^{*}+J^{*}$. Hence, by Theorem 1, either $I$ or $E$ is a subset of $I^{*}$. But each of the sets $I$ and $E$ contains a point of $J^{*}$, and $J^{*}$ contains no point of $I^{*}$. Thus we reach a contradiction, and the theorem is established.

THEOREM 5. If $J$ is a simple closed curve or triune, then $I$, the interior of $J$, contains infinitely many points.

Proof. Suppose $I$ contains exactly $n$ points, where $n$ denotes a natural number. Thus every point of $J$ is contiguous to some point of $I$. If there are infinitely many points of $J$, the closed point set $I$ contains a limit point of $J$ by Axiom C. But this is impossible since $J$ is closed. Hence $J$ contains only a finite number of points. Let $A$ and $B$ be two contiguous points of $J$, and let $X_{0}$ be a point of $J-(A+B)$. Let $X$ be a point of $I$ which is contiguous to $A$ and $Y$ a point of $I$ contiguous to $B$. Let $X Y$ denote the point $X$ or an $\operatorname{arc}$ from $X$ 
to $Y$ lying in $I$, according as $Y$ is or is not identical with $X$. The point set $X Y+A+B$ contains a simple closed curve or triune $J_{1}$ which is a subset of $J+I$ and contains $A, B$, and a point $X_{1}$ of $I$ but is such that $X_{0}$ is in the exterior of $J_{1}$. Similarly, there exists a simple closed curve or triune $J_{2}$ which is a subset of $J_{1}$ plus its interior and contains $A, B$, and a point $X_{2}$ of the interior of $J_{1}$ but is such that $X_{1}$ is in the exterior of $J_{2}$. By continuing the indicated process $n+1$ times we reach a contradiction, since $X_{1}, X_{2}, X_{3}, \cdots, X_{n-1}$ are distinct points of $I$. Hence $I$ must contain infinitely many points.

THEOREM 6. Let $J$ denote a simple closed curve whose interior I contains a point $P$ which is contiguous to at least three distinct points of $J$. Let $P_{1}, P_{2}, \cdots, P_{n},(n \geqq 3)$, be points of $J$ (in the order indicated if $n>3$ ), and let $\beta=P_{1}+P_{2}+\cdots+P_{n}$. Suppose $\beta$ is the set of all points of $J$ that are contiguous to $P$. Let $P_{k} P_{k+1},(k=1,2, \cdots, n)$, denote that arc of $J$ which contains only the points $P_{k}$ and $P_{k+1}$ of the set $\beta$, and let $P_{n+1}$ denote $P_{1}$. Let $I_{k}$, $(k=1,2, \cdots, n)$, denote the interior of the triune or simple closed curve $P+P_{k} P_{k+1}$. Then $I=P+\sum_{k=1}^{n} I_{k}$.

Proof. By Theorem $1, I_{k},(k=1,2, \cdots, n)$, is a subset of $I$. Also, if $k \neq j$, then $I_{k}$ and $I_{j}$ are mutually exclusive. Suppose $I-\left(P+\sum_{k=1}^{n} I_{k}\right)=M$, where $M$ is a non-vacuous point set, and let $M_{1}$ denote a component of $M$. Now $M$ is a domain; hence $M_{1}$ is a domain. Let $\alpha$ denote the set of all points of $J$, each of which is either a limit point of $M_{1}$ or contiguous to a point of $M_{1}$. There exists at least one point of $\alpha$. For let $Q_{1}$ denote any point of $M_{1}$, and let $Q_{1} \omega$ denote an arc from $Q_{1}$ to $\omega$ lying in the exterior of the simple closed curve or triune $P+P_{1} P_{2}$. Let $Q_{1}^{\prime}$ denote the first point that $Q_{1} \omega$ has, in order $Q_{1}$ to $\omega$, in common with $J$. Now $Q_{1}^{\prime}$ is obviously a point of $\alpha$, since $Q_{1}^{\prime}$ is the first point of $Q_{1} \omega$, in order $Q_{1}$ to $\omega$, which does not belong to $M_{1}$.

Suppose there exist two points $X$ and $Y$ of $\alpha$, which do not lie together on one of the $\operatorname{arcs} P_{1} P_{2}, P_{2} P_{3}, \cdots, P_{n} P_{1}$. Since $X$ and $Y$ are non-contiguous points of $J$, it follows that $J$ is the sum of two arcs from $X$ to $Y$ having nothing in common except their end points such that the corresponding segments of these arcs are mutually separated. There exists a point $P_{r}$ of $\beta$ on one of these segments and a point $P_{s}$ of $\beta$ on the other. Since $P_{r}$ and $P_{s}$ are both points of $\beta, P_{r}+P+P_{s}$ is an arc lying in $I+J-(M+X+Y)$. There exists an arc $P_{r} Q P_{s}$ which is a subset of $J+E-(X+Y)$, where $E$ denotes the exterior of $J$, and is such that $P_{r} Q P_{s} \cdot J$ is the sum of two mutually separated connected point sets $M_{r}$ and $M_{s}$ which contain $P_{r}$ and $P_{s}$, respectively, but no other points of $\beta$, and which separate $X$ and $Y$ on $J$. The point set $P+P_{r} Q P_{s}$ is a simple closed curve $J^{\prime}$ which contains $P$ and is such that $J^{\prime} \cdot J$ is the sum of two mutually separated connected point sets which separate $X$ and $Y$ on $J$. 
Thus $J-J^{\prime} \cdot J$ is the sum of two mutually separated segments $g_{X}$ and $g_{Y}$ which contain $X$ and $Y$, respectively. Now $J^{\prime}$ separates $X$ from $Y$, for otherwise $g_{X}$ and $g_{Y}$ would lie together in $I^{\prime}$, a complementary domain of $J^{\prime}$; therefore by Theorem 1, either $I$ or $E$ would be a subset of $I^{\prime}$, which is contrary to the fact that both $I$ and $E$ contain points of $J^{\prime}$. Since $X$ and $Y$ are boundary points of $M_{1}$ lying in different complementary domains of $J^{\prime}$, it follows that there are points of $M_{1}$ lying in different complementary domains of $J^{\prime}$. This is impossible since $M_{1}$ contains no point of $J^{\prime}$. From this contradiction we conclude that if $X$ and $Y$ are any two distinct points of $\alpha$, then there exists an arc of the set $P_{1} P_{2}, P_{2} P_{3}, \cdots, P_{n} P_{1}$ which contains both $X$ and $Y$.

Thus, if $J-\beta$ contains a point $X$ of $\alpha$, then the arc of the set $P_{1} P_{2}$, $P_{2} P_{3}, \cdots, P_{n} P_{1}$ which contains $X$ must contain all of $\alpha$. Now if $\alpha$ is not a subset of one of the arcs of the set $P_{1} P_{2}, P_{2} P_{3}, \cdots, P_{n} P_{1}$, it follows that $J-\beta$ contains no point of $\alpha$. Furthermore, it follows that $n=3$ and $\alpha=\beta=P_{1}+P_{2}+P_{3}$. In this case, since $J$ is not a triune, one of the segments $P_{1} P_{2}, P_{2} P_{3}, P_{3} P_{1}$ exists. Suppose the segment $P_{3} P_{1}$ is non-vacuous. Using methods similar to those used in the early part of the proof to get $J^{\prime}$, we obtain a simple closed curve $J^{*}$ satisfying the following conditions: (1) $J^{*}$ is a subset of $P+J+I_{3}+E$, (2) $J^{*}$ contains $P, P_{2}$, a point of the segment $P_{3} P_{1}$, a point of $E$, and a point of $I_{3}$, and (3) $J^{*} \cdot J$ is the sum of two mutually separated connected point sets which separate $P_{1}$ and $P_{3}$ on $J$, and $J-J^{*} \cdot J$ is therefore the sum of two mutually separated segments, one containing $P_{1}$ and the other containing $P_{3}$. The argument used above to show that $J^{\prime}$ separates $X$ and $Y$ may be applied here to show that $J^{*}$ separates $P_{1}$ and $P_{3}$. Hence $J^{*}$ separates two points $P_{1}^{\prime}$ and $P_{3}^{\prime}$ of $M_{1}$, since $P_{1}$ and $P_{3}$ are points of $\alpha$. But this is impossible since $M_{1}$ is connected and contains no point of $J^{*}$. Thus we reach a contradiction, and we conclude that there exists an arc $P_{i} P_{i+1}$ of the set of $\operatorname{arcs} P_{1} P_{2}, P_{2} P_{3}, \cdots, P_{n} P_{1}$ which contains $\alpha$.

Let $E_{i}$ denote the exterior of the simple closed curve or triune $\left(P+P_{i} P_{i+1}\right)$. The domain $E_{i}$ contains $M_{1}$, and we write $E_{i}=M_{1}+\left(E_{i}-M_{1}\right)$. The boundary of $M_{1}$ is a subset of $\left(P+P_{i} P_{i+1}\right)$. Hence $M_{1}$ and $E_{i}-M_{1}$ are mutually separated, contrary to Axiom 3. Thus we reach a contradiction, and the theorem is established.

Theorem 7. Let $P_{1}, P_{2}, \cdots, P_{n},(n \geqq 3)$, be points of the simple closed curve $J$ (in the order indicated if $n>3)$. Let $A_{1}, A_{2}, \cdots, A_{k},(k \geqq 2)$, be points of the arc $A_{1} A_{k}$ (in the order indicated if $k>2$ ) where $A_{1} A_{k}$ is a subset of $I$, the interior of $J$. Let $\beta=P_{1}+P_{2}+\cdots+P_{n}$, and let $\gamma=A_{1}+A_{2}+\cdots+A_{k}$. Suppose $\beta$ is the set of all points of $J$ each of which is contiguous to at least one point of $A_{1} A_{k}$, and suppose $\gamma$ is the set of all points of $A_{1} A_{k}$ each of which is contiguous to at least one point of $\beta$. Suppose furthermore that no point of $\beta$ is contiguous to both 
$A_{1}$ and $A_{k}$, but that $P_{1}$ is contiguous to $A_{1}$ and to no other point of $\gamma$ and $P_{n}$ is contiguous to $A_{k}$ and to no other point of $\gamma$. It follows that if $j<j^{\prime}$, and if $P_{j}$ is contiguous to $A_{i}$ and $P_{j^{\prime}}$ is contiguous to $A_{i^{\prime}}$, then $i \leqq i^{\prime}$. Let $P_{j} P_{j+1}$, $(j=1,2, \cdots, n)$, denote that arc of $J$ from $P_{j}$ to $P_{j+1}$ which contains no other point of $\beta$, where $P_{n+1}$ denotes $P_{1}$. Let $A_{i} A_{i+1},(i=1,2, \cdots, k-1)$, denote the arc of $A_{1} A_{k}$ from $A_{i}$ to $A_{i+1}$. Let $I_{j},(j=1,2, \cdots, n-1)$, denote the interior of $C_{j}$, where $C_{j}$ denotes the triune or simple closed curve $\left(P_{j} P_{j+1}+A_{i}\right)$ in case $P_{j}$ and $P_{j+1}$ are both contiguous to $A_{i}$, or $C_{j}$ denotes the simple closed curve $\left(P_{j} P_{j+1}+A_{i} A_{i+1}\right)$ in case no point of $\gamma$ is contiguous to both $P_{j}$ and $P_{i+1}$, but where $P_{j}$ is contiguous to $A_{i}$ and $P_{j+1}$ is contiguous to $A_{i+1}$. Let $I_{n}$ denote the interior of the simple closed curve $\left(P_{n} P_{1}+A_{1} A_{k}\right)$. If $P_{j}$ is contiguous to each of the points $A_{i_{j}}, A_{i_{j+1}}, \cdots, A_{i_{j+k}}$, let $I_{j 1}, I_{j 2}, \cdots, I_{j k_{j}}$ denote the interiors of $\left(P_{j} \nmid A_{i_{j}} A_{i_{j+1}}\right),\left(P_{j}+A_{i_{j+1}} A_{i_{j}+2}\right), \cdots$, and $\left(P_{j}+A_{i_{j+k}+1} A_{i_{j}+k_{j}}\right)$, respectively. Then

$$
I=A_{1} A_{k}+\sum_{j=1}^{n} I_{j}+\sum_{j=1}^{n} \sum_{t=1}^{k_{j}} I_{j t},
$$

where $I_{j t}$ is a null set if $P_{j}$ is contiguous to only one point of $\gamma$.

The theorem is illustrated by Fig. 1 .

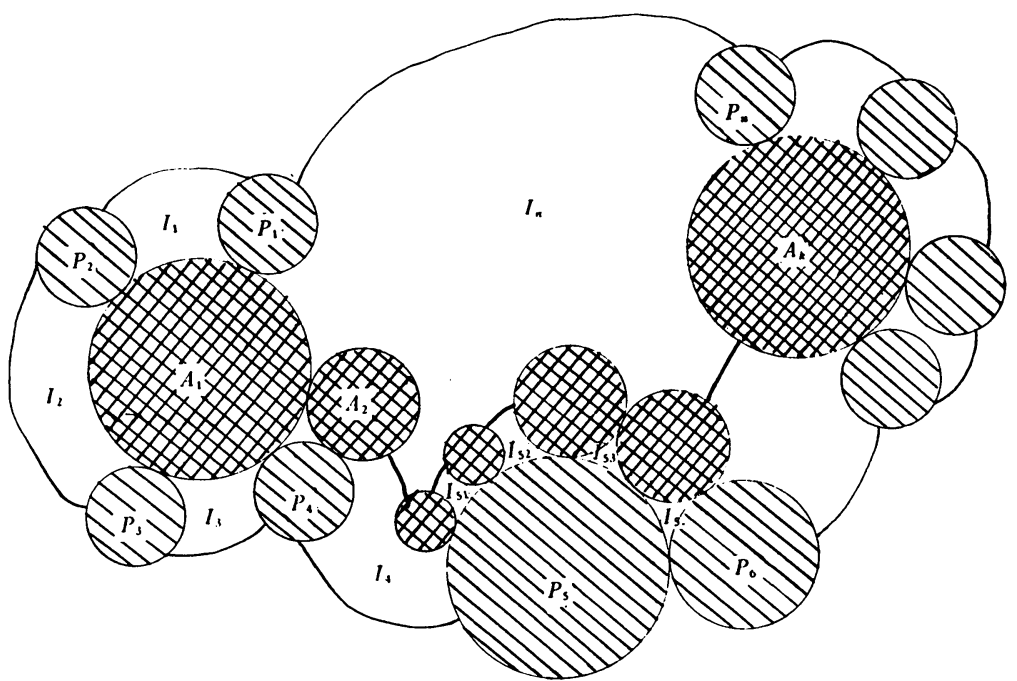

FIG. 1

Proof. First I shall prove the assertion made in the statement of the theorem to the effect that if $j<j^{\prime}$, and if $P_{j}$ is contiguous to $A_{i}$ and $P_{j^{\prime}}$ is contiguous to $A_{i^{\prime}}$, then $i \leqq i^{\prime}$. Suppose there exist two integers $j$ and $j^{\prime}$ such that 
$j<j^{\prime}, P_{j}$ and $P_{j^{\prime}}$ are contiguous to $A_{i}$ and $A_{i^{\prime}}$, respectively, and $i>i^{\prime}$. It follows that $i>1$ and $i^{\prime}<k$. Also since $P_{1}$ is contiguous to $A_{1}$ but to no other point of $\gamma$, we see that $1<j$. Again, since $P_{n}$ is contiguous to $A_{k}$ but to no other point of $\gamma$, we see that $j^{\prime}<n$. Thus $1<j<j^{\prime}<n$; hence $P_{1}$ and $P_{j^{\prime}}$, separate $P_{j}$ and $P_{n}$ on $J$. Let $A_{i_{1}}$ be the point of $\gamma$ with lowest subscript which is contiguous to $P_{j^{\prime}}$, and let $A_{i_{1}}$ be the point of $\gamma$ with the highest subscript which is contiguous to $P_{j}$. Let $A_{1} A_{i_{1}}{ }^{\prime}$ denote the arc $A_{1} A_{i_{1}}$ of $A_{1} A_{k}$ or the point $A_{1}$ according as $i_{1}^{\prime}$ is not or is 1 , and let $A_{i_{1}} A_{k}$ denote the $\operatorname{arc} A_{i_{1}} A_{k}$ of $A_{1} A_{k}$ or the point $A_{k}$ according as $i_{1}$ is not or is $k$. Now $P_{1}+A_{1} A_{i_{1}}+P_{j^{\prime}}$ and $P_{j}+A_{i_{1}} A_{k}+P_{n}$ are arcs satisfying the conditions of Theorem 4 . Hence $A_{1} A_{i_{1}{ }^{\prime}}$ and $A_{i_{1}} A_{k}$ have a point in common. But $1 \leqq i_{1}^{\prime} \leqq i^{\prime}<i \leqq i_{1} \leqq k$; therefore $A_{1} A_{i_{1}}$ and $A_{i_{1}} A_{k}$ cannot have a point in common. Thus we reach a contradiction, and we conclude that if $j<j^{\prime}$, and if $P_{j}$ and $P_{j^{\prime}}$ are contiguous to $A_{i}$ and $A_{i^{\prime}}$, respectively, then $i \leqq i^{\prime}$.

This result enables us to verify the following implications made in the statement of the theorem: (1) there do not exist two distinct points of $\gamma$ each of which is contiguous to both $P_{j}$ and $P_{j+1}$; (2) if no point of $\gamma$ is contiguous to both $P_{j}$ and $P_{j+1}$, then there exists an integer $i$ such that $A_{i}$ is contiguous to $P_{j}$ and $A_{i+1}$ is contiguous to $P_{j+1}$; and (3) if no point of $\beta$ is contiguous to both $A_{i}$ and $A_{i+1}$, then there exists an integer $j$ such that $j<n$ and $P_{j}$ and $P_{j+1}$ are contiguous to $A_{i}$ and $A_{i+1}$, respectively.

Suppose $I-\left(A_{1} A_{k}+\sum_{j=1}^{n} I_{j}+\sum_{j=1}^{n} \sum_{t=1}^{k_{j}} I_{j t}\right)=M$ is a non-vacuous point set. Let $M_{1}$ be a component of the domain $M$, and let $\alpha$ denote the boundary of $M_{1}$. Obviously $\alpha$ is a subset of $J+A_{1} A_{k}$. Furthermore, $\alpha$ contains at least one point of $J$ and at least one point of $A_{1} A_{k}$. For let $P$ be a point of $M_{1}$, and let $P \omega$ be an arc from $P$ to $\omega$ lying in the exterior of $\left(A_{1} A_{k}+P_{n} P_{1}\right)$. Now $P \omega$ obviously contains a point of $\alpha \cdot J$. Similarly, let $P A_{1}$ denote an arc from $P$ to $A_{1}$ which lies in $I$. Now $P A_{1}$ contains a point of $\alpha \cdot A_{1} A_{k}$.

Suppose $J$ contains two non-contiguous points $X$ and $Y$ of $\alpha$. If $X$ and $Y$ do not lie together on any one of the $\operatorname{arcs} P_{1} P_{2}, P_{2} P_{3}, \cdots, P_{n} P_{1}$, there exist two points $P_{r}$ and $P_{s}$ of $\beta$ which separate $X$ and $Y$ on $J$; hence there exists a simple closed curve $J^{\prime}$ such that: (1) $J^{\prime}$ is a subset of $E+J+A_{1} A_{k}$; (2) $J^{\prime} \cdot J=M_{r}+M_{s}$ where $M_{r}$ and $M_{s}$ are two mutually separated continua which contain $P_{r}$ and $P_{s}$, respectively, and which separate $X$ and $Y$ on $J$; therefore $J-J^{\prime} \cdot J=g_{X}+g_{Y}$ where $g_{X}$ and $g_{Y}$ are mutually separated segments containing $X$ and $Y$, respectively; (3) $J^{\prime}$ contains at least one point $O$ of $E$ and at least one point of $A_{1} A_{k}$. If $X$ and $Y$ lie together on the $\operatorname{arc} P_{j} P_{j+1}$ of the set $P_{1} P_{2}, P_{2} P_{3}, \cdots, P_{n} P_{1}$, there exists a simple closed curve $J^{\prime}$ such that: (1) $J^{\prime}$ is a subset of $E$ plus the segment $X Y$ of $P_{j} P_{j+1}+I_{j}+A_{1} A_{k}+\left(J-P_{j} P_{j+1}\right)$; (2) $J^{\prime} \cdot J$ is the sum of two mutually separated continua which separate $X$ 
and $Y$ on $J$; hence $J-J^{\prime} \cdot J=g_{X}+g_{Y}$ where $g_{X}$ and $g_{Y}$ are mutually separated segments containing $X$ and $Y$, respectively; (3) $J^{\prime}$ contains at least one point $O$ of $E$ and at least one point of $A_{1} A_{k}$. Now, in either case it may be readily shown that $J^{\prime}$ separates $X$ from $Y$ and hence that $J^{\prime}$ separates two points $X^{\prime}$ and $Y^{\prime}$ of $M_{1}$. But this is impossible since $M_{1}$ is connected and contains no point of $J^{\prime}$. From this contradiction we conclude that $\alpha \cdot J$ consists either of a single point or of two contiguous points. By an analogous argument it may be shown that $\alpha \cdot A_{1} A_{k}$ consists either of a single point or of two contiguous points.

Now I wish to show that $\alpha$ is a subset of a simple closed curve or triune $J^{*}$, which is a subset of $J+A_{1} A_{k}$, satisfying the condition that either there exists an integer $j$ such that $I_{j}$ is the interior of $J^{*}$, or there exists a pair of integers $(j, t)$ such that $I_{j t}$ is the interior of $J^{*}$. If $\alpha \cdot J$ is a subset of $P_{n} P_{1}$, then $J^{*}=P_{n} P_{1}+A_{1} A_{k}$. If $\alpha \cdot J$ is not a subset of $P_{n} P_{1}$, we shall use the following procedure.

Case 1. Suppose that $P_{j} P_{j+1},(j \neq n)$, is the only arc of the set $P_{1} P_{2}$, $P_{2} P_{3}, \cdots, P_{n} P_{1}$ which contains $\alpha \cdot J$. It follows that if $X$ denotes either $P_{j}$ or $P_{j+1}$, then $P_{j} P_{j+1}-X$ contains at least one point $Z$ of $\alpha$. If $P_{j}$ and $P_{j+1}$ are both contiguous to the point $A_{i}$ of $\gamma$, then we may show that $A_{i}=\alpha \cdot A_{1} A_{k}$. Suppose the contrary. Let $Y$ denote a point of $\alpha \cdot A_{1} A_{k}$ which is different from $A_{i}$. Now $Y$ either precedes or follows $A_{i}$, in the order $A_{1}$ to $A_{k}$. Suppose $Y$ precedes $A_{i}$. Then $i \neq 1$ and $j \neq 1$, and $Y$ is not contiguous to $P_{j+1}$. There exists a simple closed curve $J^{\prime}$ having the following properties: (1) $J^{\prime}$ is a subset of $J+A_{1} A_{k}+I_{n}$; (2) $J^{\prime} \cdot J$ is a connected point set such that the connected point set $J-J^{\prime} \cdot J$ contains $P_{n}+\left(P_{j} P_{j+1}-P_{j}\right)$; (3) $J^{\prime} \cdot A_{1} A_{k}$ is a connected set such that $A_{1} A_{k}-J^{\prime} \cdot A_{1} A_{k}$ is either a connected set containing $Y$ and $A_{1}$, or the sum of two mutually separated connected sets, one containing $Y$ and $A_{1}$ and the other containing $A_{k}$; (4) $J^{\prime}$ contains at least one point of $I_{n}$ and at least one point of $J-P_{n} P_{1}$. By means of these four conditions imposed on $J^{\prime}$ we may readily show that $J^{\prime}$ separates $Y$ from $Z$ and hence that $J^{\prime}$ separates two points $Y^{\prime}$ and $Z^{\prime}$ of $M_{1}$. But this is impossible since $M_{1}$ is connected and contains no point of $J^{\prime}$. Thus we conclude that $Y$ cannot precede $A_{i}$ on $A_{1} A_{k}$. Similarly $Y$ cannot follow $A_{i}$ on $A_{1} A_{k}$. Therefore $A_{i}=\alpha \cdot A_{1} A_{k}$. Thus $\left(A_{i}+P_{j} P_{j+1}\right)$ is a simple closed curve or triune $J^{*}$ which contains $\alpha$ and whose interior is $I_{j}$.

If there exists no point of $\gamma$ which is contiguous to both $P_{j}$ and $P_{j+1}$, then there exists an integer $i$ such that $P_{j}$ is contiguous to $A_{i}$ and $P_{j+1}$ is contiguous to $A_{i+1}$. In this case we show that $\alpha \cdot A_{1} A_{k}$ is a subset of $A_{i} A_{i+1}$. Suppose the contrary. Let $Y$ denote a point of $\alpha \cdot A_{1} A_{k}$ not on $A_{i} A_{i+1}$. Either $Y$ precedes $A_{i}$ or $Y$ follows $A_{i+1}$, in the order $A_{1}$ to $A_{k}$. Suppose $Y$ precedes $A_{i}$. 
Then $i \neq 1$ and $j \neq 1$ or $n$. Now there exists a simple closed curve $J^{\prime}$ having the following properties: (1) $J^{\prime}$ is a subset of $J+A_{1} A_{k}+I_{n}$; (2) $J^{\prime} \cdot J$ is a connected point set such that the connected set $J-J^{\prime} \cdot J$ contains $P_{n}+\left(P_{j} P_{j+1}-P_{j}\right) ;(3) J^{\prime} \cdot A_{1} A_{k}$ is connected, and $A_{1} A_{k}-J^{\prime} \cdot A_{1} A_{k}$ is the sum of two mutually separated connected point sets, one containing $Y$ and $A_{1}$, and the other containing $A_{i+1}$ and $A_{k}$; (4) $J^{\prime}$ contains at least one point of $I_{n}$ and at least one point of $J-P_{n} P_{1}$. Thus again $J^{\prime}$ separates $Y$ from $Z$ and hence separates two points $Y^{\prime}$ and $Z^{\prime}$ of $M_{1}$. Again we reach a contradiction, and we conclude that $Y$ cannot precede $A_{i}$. The same argument applies to show that $Y$ cannot follow $A_{i+1}$. Hence $A_{1} A_{k}$ is a subset of $A_{i} A_{i+1}$, and $\left(P_{j} P_{j+1}+A_{i} A_{i+1}\right)$ is therefore a simple closed curve $J^{*}$ containing $\alpha$. The interior of $J^{*}$ is $I_{j}$.

Case 2. If there exists no integer $r$ such that $P_{r} P_{r+1}$ is the only arc of the set $P_{1} P_{2}, P_{2} P_{3}, \cdots, P_{n} P_{1}$ which contains $\alpha \cdot J$, it follows that $\alpha \cdot J$ is a point $P_{j}$ of $\beta$. The case in which $\alpha \cdot J=P_{n}$ has been disposed of in the paragraph preceding Case 1 . Hence suppose $j<n$. Let $A_{i}$ be the point of $\gamma$ with lowest subscript which is contiguous to $P_{j}$, and let $A_{i}{ }^{\prime}$ be the point of $\gamma$ with highest subscript which is contiguous to $P_{j}$. By means of an argument like that used in Case 1, it may be shown that no point of $\alpha \cdot A_{1} A_{k}$ precedes $A_{i}$ or follows $A_{i}{ }^{\prime}$ in the order from $A_{1}$ to $A_{k}$. If $i=i^{\prime}$, then $\alpha \cdot A_{1} A_{k}=A_{i}$. Thus $\left(P_{j} P_{j+1}+A_{i}\right)$ or $\left(P_{j} P_{j+1}+A_{i} A_{i+1}\right)$, according as $P_{j}$ and $P_{j+1}$ are or are not both contiguous to $A_{i}$, is a triune or simple closed curve $J^{*}$ which contains $\alpha$ and whose interior is $I_{j}$. If $i \neq i^{\prime}$, then the $\operatorname{arc} A_{i} A_{i}{ }^{\prime}$ of $A_{1} A_{k}$ contains $\alpha \cdot A_{1} A_{k}$. Furthermore, since $\alpha \cdot A_{1} A_{k}$ consists either of a single point or of two contiguous points, it follows that $\alpha \cdot A_{1} A_{k}$ is a subset of one of the arcs of the set $A_{i} A_{i+1}, A_{i+1} A_{i+2}, \cdots, A_{i^{\prime}-1} A_{i^{\prime}}$. Suppose the $\operatorname{arc} A_{i+t-1} A_{i+t} \operatorname{contains} \alpha \cdot A_{1} A_{k}$. Then since $A_{s},\left(i \leqq s \leqq i^{\prime}\right)$, is contiguous to $P_{j}$, it follows that $\left(P_{j}+A_{i+t-1} A_{i+t}\right)$ is a simple closed curve or triune $J^{*}$ which contains $\alpha$ and whose interior is $I_{j t}$. Thus for any case it has been shown that $\alpha$ is a subset of a simple closed curve or triune $J^{*}$ satisfying the condition that either there exists an integer $j$ such that $I_{j}$ is the interior of $J^{*}$, or there exists a pair of integers $(j, t)$ such that $I_{j t}$ is the interior of $J^{*}$.

Let $E^{*}$ denote the exterior of $J^{*}$. Now $E^{*}$ contains $M_{1}$; hence $E^{*}=M_{1}$ $+\left(E^{*}-M_{1}\right)$, where both $M_{1}$ and $E^{*}-M_{1}$ are non-vacuous point sets neither of which contains a point of $\alpha$. Thus $E^{*}$ is the sum of two mutually separated sets, contrary to Axiom 3; and the theorem is established.

THEOREM 8. Let the following changes, and none other, be made in the hypotheses of Theorem 7: $A_{1}, A_{2}, \cdots, A_{k},(k \geqq 1)$, are points of an arc $A_{1} T$ (in the order indicated if $k>1$ ) where $A_{1} T-T$ is a subset of $I$ and $T$ is a point of 
the segment $P_{n} P_{1},(n \geqq 2)$. The set $\gamma=A_{1}+A_{2}+\cdots+A_{k}$ is the set of all points of $A_{1} T-T$ which are contiguous to points of $J ; \beta=P_{1}+P_{2}+\cdots+P_{n}$ is the set of all points of $J$ which are contiguous to points of $\gamma$. The point $P_{n}$ is no longer restricted to be contiguous to $A_{k}$ alone of the set $\gamma ; I_{n}$ denotes the interior of $P_{n} T+A_{k} T ;$ and $I_{0}$ denotes the interior of $A_{1} T+T P_{1}$. Then

$$
I=\left(A_{1} T-T\right)+\sum_{j=0}^{n} I_{j}+\sum_{j=1}^{n} \sum_{t=1}^{k_{j}} I_{j \iota} .
$$

If $\beta=P_{1}+P_{2}+P_{3}+\cdots$ and $\gamma=A_{1}+A_{2}+A_{3}+\cdots$, where $\beta$ and $\gamma$ are infinite sets each having $T$ as its only limit point, then

$$
I=\left(A_{1} T-T\right)+\sum_{j=0}^{\infty} I_{j}+\sum_{j=1}^{\infty} \sum_{t=1}^{k_{j}} I_{j t} .
$$

THEOREM 9. Let the following changes, and none other, be made in the hypotheses of Theorem 7: V, $A_{1}, A_{2}, \cdots, A_{k}, T,(k \geqq 1)$, are points of the arc $V A_{1} T$ in the order indicated, where the segment $V A_{1} T$ is a subset of $I$, and where $V$ and $T$ are points of $J$ in the order $V, P_{1}, P_{2}, \cdots, P_{n}, T,(n \geqq 1)$. The set $\gamma=A_{1}+A_{2}+\cdots+A_{k}$ is the set of all points of the segment $V A_{1} T$ which are contiguous to points of $J$, and $\beta=P_{1}+P_{2}+\cdots+P_{n}$ is the set of all points of $J$ which are contiguous to points of $\gamma$. The points $P_{1}$ and $P_{n}$ are no longer restricted as to the number of points of $\gamma$ to which they are contiguous. If $I_{0}, I_{n}$, and $I^{*}$ denote the interiors of $\left(V P_{1}+V A_{1}\right),\left(P_{n} T+A_{k} T\right)$, and $\left(T V+V A_{1} T\right)$, respectively, where $T V$ is that arc of $J$ not containing $P_{1}$, then

$$
I=\text { segment } V A_{1} T+I^{*}+\sum_{j=0}^{n} I_{j}+\sum_{j=1}^{n} \sum_{i=1}^{k_{j}} I_{j t} \text {. }
$$

If $\beta=P_{1}+P_{2}+P_{3}+\cdots$ and $\gamma=A_{1}+A_{2}+A_{3}+\cdots$ are infinite sets each having $T$ as its only limit point, then

$$
I=\text { segment } V A_{1} T+I^{*}+\sum_{j=0}^{\infty} I_{j}+\sum_{j=1}^{\infty} \sum_{t=1}^{k_{i}} I_{j t} .
$$

A similar formula holds for the case in which each of the points $V$ and $T$ is approached sequentially by a sequence from $\beta$ and by a sequence from $\gamma$.

THEOREM 10. Let the following changes be made in the hypotheses of Theorem 7: $P_{1}$ is contiguous to both $A_{1}$ and $A_{k}$ but to no other point of $\gamma$. Either each of the points $A_{1}$ and $A_{k}$ is contiguous to some point of $\beta-P_{1}$ or else $k>2$. Then either it is true that if $P_{j}$ and $P_{j^{\prime}}$ are contiguous to $A_{i}$ and $A_{i^{\prime}}$, respectively, and if $j<j^{\prime}$, then $i \leqq i^{\prime}$, or it is true that if $P_{j}$ and $P_{j^{\prime}}$ are contiguous to $A_{i}$ and $A_{i^{\prime}}$, respectively, and if $j<j^{\prime}$, then $i \geqq i^{\prime}$. If the former condition holds, if $I^{*}$ denotes 
the interior of $A_{1} A_{k}+P_{1}$, and if $I_{n}$ denotes the point set obtained by substituting $n$ for $j$ in the definition of $I_{j}$ given in the statement of Theorem 7 , then

$$
I=A_{1} A_{k}+I^{*}+\sum_{j=1}^{n} I_{j}+\sum_{j=2}^{n} \sum_{t=1}^{k_{j}} I_{j t} .
$$

The theorem is illustrated by Fig. 2 .
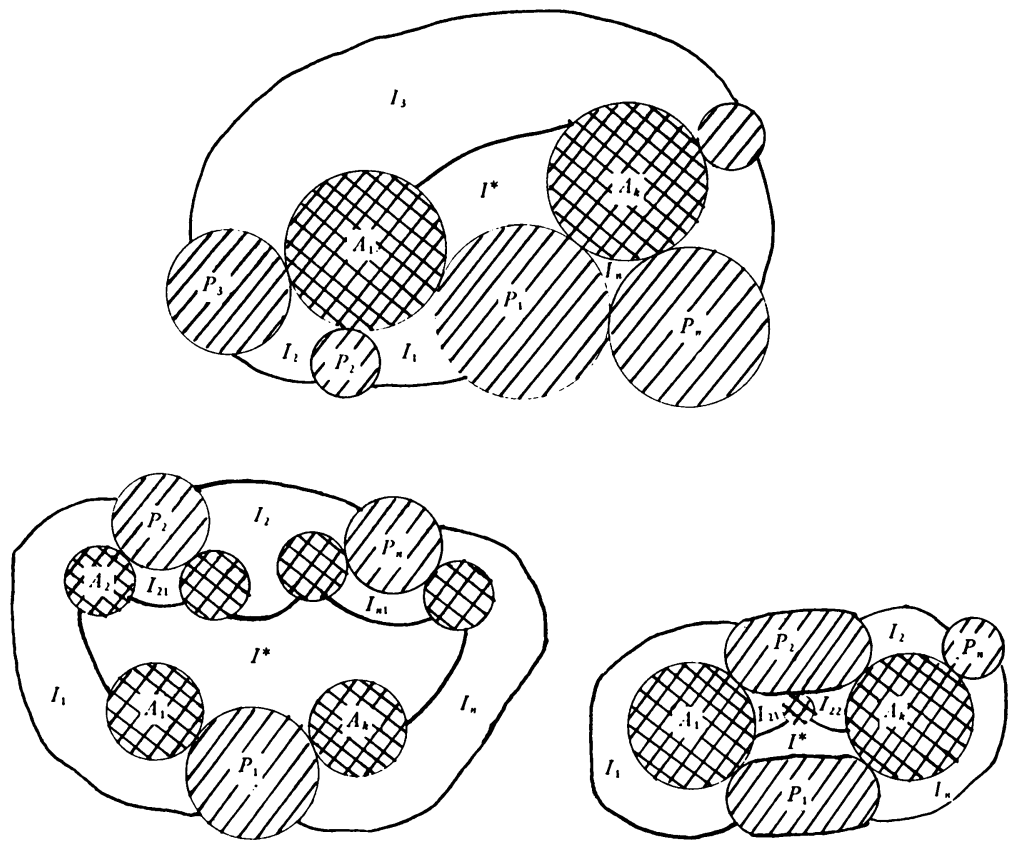

FIG. 2

If in Theorem 10, $\beta=P_{1}+P_{2}$, where $P_{1}$ and $P_{2}$ are non-contiguous, then $J$ is the sum of two arcs, $P_{1} X P_{2}$ and $P_{1} Y P_{2}$, and is such that the corresponding segments are mutually separated. Suppose $k \geqq 3$. (1) If at least one of the points $A_{1}$ and $A_{k}$, say $A_{1}$, is contiguous to $P_{2}$, then by Theorem 3, $I-A_{1}=D_{1}+D_{2}$, where $D_{1}$ and $D_{2}$ are the interiors of the simple closed curves $P_{1} X P_{2}+A_{1}$ and $P_{1} Y P_{2}+A_{1}$, respectively. Let $I_{1}$ denote that domain above which does not contain $A_{k}$. Suppose $I_{1}=D_{1}$. Let $I_{2}$ denote the interior of $P_{1} Y P_{2}+A_{k}$ or of $P_{1} Y P_{2}+A_{k-1} A_{k}$, according as $A_{k}$ is or is not contiguous to $P_{2}$. (2) If neither $A_{1}$ nor $A_{k}$ is contiguous to $P_{2}$, then $I-A_{1} A_{2}=D^{*}+D^{* *}$, where $D^{*}$ and $D^{* *}$ are the interiors of $P_{1} X P_{2}+A_{1} A_{2}$ and $P_{1} Y P_{2}+A_{1} A_{2}$, respectively. Let $I_{1}$ denote the domain above which does not contain $A_{k}$. Suppose $I_{1}=D^{*}$. Let $I_{2}$ denote the interior of $P_{1} Y P_{2}+A_{k-1} A_{k}$. Then in either of 
these two cases we have, in accordance with the above notation, the following theorem:

Theorem 11. $I=A_{1} A_{k}+I^{*}+I_{1}+I_{2}+\sum_{t=1}^{k_{2}} I_{2}$.

This theorem is illustrated by Fig. 3 .
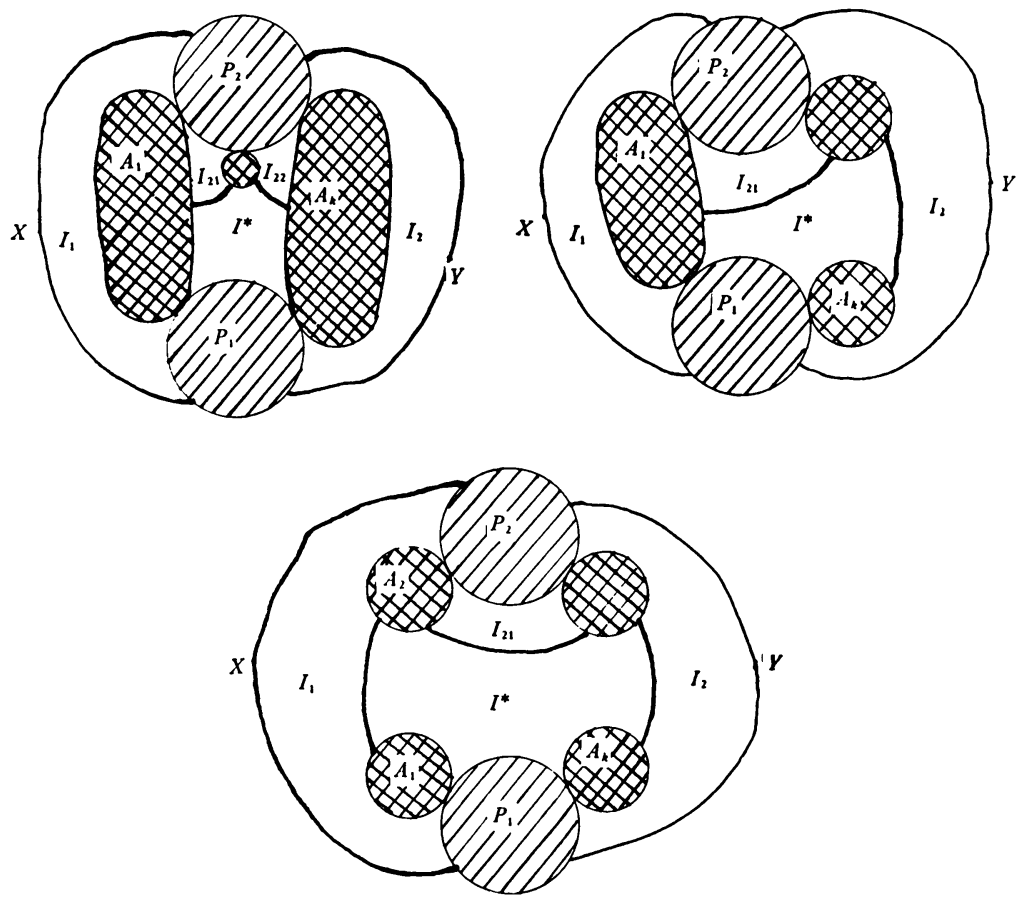

Fig. 3

TheOREM 12. Let $J$ and $C$ denote two point sets, each of which is either a simple closed curve or a triune. Let $I$ and $D$ denote the interiors of $J$ and $C, r e-$ spectively, and suppose $P$ is a point of $I \cdot D$. Then there exists a simple closed curve or triune $Q$ such that (1) $Q$ is a subset of $J+C$, and (2) the interior of $Q$ contains $P$ and is a subset of $I \cdot D$.

Indication of proof. If at least one of the two sets $J$ and $C$ is a triune, one of the sets is a subset of the other plus its interior and hence will have the properties required of $Q$.

A similar situation exists if both $J$ and $C$ are simple closed curves and one of them is a subset of the other plus its interior.

If neither of the simple closed curves $J$ and $C$ is a subset of the other plus its interior, let $A$ be a point of $J \cdot D$, and let $P A$ be an $\operatorname{arc}$ from $P$ to $A$ lying 
wholly in $D$. Let $O$ denote the first point, in the order $P$ to $A$, that $P A$ has in common with $J$. I wish to show the existence of a simple closed curve or triune $Q^{\prime}$ such that (1) $Q^{\prime}$ is a subset of $C+J \cdot D$, (2) $Q^{\prime} \cdot C$ is connected, (3) $Q^{\prime} \cdot J \cdot D$ is connected and contains $O$, and (4) the interior of $Q^{\prime}$ contains $(P O-O)$. Obviously, the existence of $Q^{\prime}$ will be established if it can be shown that there exists a point set $K$ (which shall contain $Q^{\prime} \cdot J \cdot D$ ) which is a connected subset of $J$ containing $O$ and which satisfies with respect to $C$ those conditions satisfied by any one of the point sets $P, A_{1} A_{k}$, segment $A X B$, $\left(A_{1} T-T\right)$, segment $V A_{1} T, A_{1} A_{k}$, or $A_{1} A_{k}$ with respect to the corresponding given simple closed curve of Theorems $6,7,3,8,9,10$, and 11, respectively. There are several cases.

Case 1. Suppose $O$ is not contiguous to any point of $C$. Let $B$ denote a point of $J \cdot E$. The set $J$ is the sum of two arcs from $O$ to $B$, say $O X B$ and $O Y B$. Let $M$ denote the closed point set consisting of $C$ together with all points $Z$ of $D$ such that $Z$ is contiguous to at least one point of $C$. Let $A_{1}$ and $A_{2}$ denote the first points that $O X B$ and $O Y B$ have in common with $M$, in the order $O$ to $B$. If both $A_{1}$ and $A_{2}$ are points of $C$, the hypothesis of Theorem 3 is satisfied, and the segment $A_{1} O A_{2}$ is the desired point set $K$.

Case 2. Suppose $O$ is not contiguous to any point of $C$ and that one of the points $A_{1}$ and $A_{2}$ described in Case 1 , say $A_{1}$, is a point of $D$, while the other, $A_{2}$, is a point of $C$. If $A_{1}$ is contiguous to two or more points of $C$, then the hypotheses of Theorem 8 are satisfied, and $\left(A_{1} O A_{2}-A_{2}\right)$ is the desired point set $K$. If $A_{1}$ is contiguous to only one point $F$ of $C$, and $F$ is not contiguous to $A_{2}$, then the hypotheses of Theorem 3 are satisfied, and $\left(A_{1} O A_{2}-A_{2}\right)$ is the desired point set $K$. If $F$ is contiguous to $A_{2}$, let $A_{1}^{\prime}$ denote the first point of $O X B$, in the order $O$ to $B$, which is either a point of $D$ that is contiguous to a point of $C-F$ or else a point of $C$. Now if $A_{1}^{\prime}$ is a point of $D$, the hypothesis of Theorem 8 is satisfied, and $\left(A_{1}^{\prime} O A_{2}-A_{2}\right)$ is the desired point set $K$. If $A_{1}^{\prime}$ is a point of $C$, the hypothesis of Theorem 9 is satisfied, and the segment $A_{1} O A_{2}$ is the desired point set $K$.

The remaining cases may be treated with similar methods.

If $Q^{\prime}$ is a triune, $Q^{\prime}$ has the properties required of $Q$, since the interior of $Q^{\prime}$ cannot contain any points of $J$. (No simple closed curve may contain a point of each complementary domain of a triune.)

If $Q^{\prime}$ is a simple closed curve whose interior contains no point of $J$, then $Q^{\prime}=Q$. If the interior of $Q^{\prime}$ contains any points of $J$, let $M$ denote the set of all such points of $J$. Now, $M+Q^{\prime}$ contains a simple closed curve $Q$ having the required properties. The proof of this last statement is little different from the proof for spaces in which there do not exist contiguous points.

As an immediate result of Theorem 12 we have the following theorem: 
TheOREM 13. Let $J_{1}, J_{2}, \cdots, J_{n}$ denote $n$ point sets each of which is either a simple closed curve or a triune; and suppose that $I_{1}, I_{2}, \cdots, I_{n}$, the interiors of $J_{1}, J_{2}, \cdots, J_{n}$, respectively, have a point $O$ in common. Then there exists a simple closed curve or triune $J$ which is a subset of $J_{1}+J_{2}+\cdots+J_{n}$ and is such that $I$, the interior of $J$, is a subset of $I_{1} \cdot I_{2} \cdots I_{n}$ and contains $O$.

Theorem 14. Suppose each of the sets $J_{1}$ and $J_{2}$ is either a simple closed curve or a triune. Let $I_{1}$ and $I_{2}$ denote the interiors of $J_{1}$ and $J_{2}$, respectively, and suppose $I_{1}+I_{2}$ is connected. Then there exists a simple closed curve or triune $J$ which is a subset of $J_{1}+J_{2}$ and whose interior is a subset of $I_{1} \cdot I_{2}$.

Indication of proof. Assume the theorem false. Then by Theorem 12 $I_{1} \cdot I_{2}=0$. Hence there exist points $P_{1}$ and $P_{2}$ of $I_{1}$ and $I_{2}$, respectively, such that $P_{1}$ is contiguous to $P_{2}$. Thus $P_{1}$ is a point of $J_{2}$ and $P_{2}$ is a point of $J_{1}$. There exists a point $F$ of $J_{1} \cdot J_{2}$ and an arc $P_{1} F$ of $J_{2}$ such that $P_{1} F-F$ is a subset of $I_{1}$. Let $P_{2} F$ denote an arc of $J_{1}$. The point set $P_{1} F+P_{2} F$ contains a simple closed curve or triune $J^{\prime}$ containing $P_{1}$ and $P_{2}$, whose interior $I^{\prime}$ is a subset of $I_{1}$. The set $J^{\prime}+I^{\prime} \cdot J_{2}$ contains a simple closed curve or triune $J$ satisfying the required conditions. Thus the assumption is false, and the theorem is established.

With the help of Theorems 12 and 14, the following theorem may be established:

THEOREM 15. Let $J_{1}$ and $J_{2}$ denote two point sets, each of which is either a simple closed curve or a triune. Let $I_{1}$ and $I_{2}$ denote the interiors of $J_{1}$ and $J_{2}$, respectively. Suppose $I_{1}+I_{2}$ is connected. Then there exists a simple closed curve or triune $J$ which is a subset of $J_{1}+J_{2}$, and whose interior contains $I_{1}+I_{2}$.

ThEOREM 16. Let $J_{1}, J_{2}, \cdots, J_{n}$ denote $n$ point sets each of which is either a simple closed curve or a.triune. Let $I_{1}, I_{2}, \cdots, I_{n}$ denote the interiors of $J_{1}, J_{2}, \cdots, J_{n}$, respectively. Suppose $I_{1}+I_{2}+\cdots+I_{n}$ is connected. Then there exists a point set $J$ which is either a simple closed curve or a triune, which is a subset of $J_{1}+J_{2}+\cdots+J_{n}$, and which is such that $I$, the interior of $J$, contains $I_{1}+I_{2}+\cdots+I_{n}$.

THEOREM 17. If $J$ is a simple closed curve or triune, then $I$, the interior of $J$, contains at least one point which is not contiguous to any point of $J$.

Proof. Suppose each point of $I$ is contiguous to some point of $J$. Let $P$ be any point of $I$. I wish to show the existence of two distinct points of $I$, each contiguous to $P$. Since $I$ is connected and contains infinitely many points, $P$ is either contiguous to a point of $I$ or is a limit point of $I$. By Axiom C, $J$ contains all limit points of $I$; hence $P$ must be contiguous to a point $Q$ of $I$. In case there exists a point $P_{1}$ of $J$ which is contiguous to both 
$P$ and $Q$, then $I_{1}$, the interior of the triune $P Q P_{1}$, is a connected subset of $I$. Since $J$ contains all the limit points of $I_{1}$, it follows that $P$ is contiguous to a point $T$ of $I_{1}$. Thus for this case there exist two distinct points $(Q$ and $T$ ) of $I$, each contiguous to $P$. In case no point of $J$ is contiguous to both $P$ and $Q$, let $P_{1}$ and $Q_{1}$ denote points of $J$ which are contiguous to $P$ and $Q$, respectively, and let $P_{1} Q_{1}$ denote an arc of $J$. The point set $P_{1} Q_{1}+P+Q$ contains a simple closed curve $J_{2}$, which contains $P$ and $Q$. Let $I_{2}$ denote the interior of $J_{2}$. The argument used above may be used here to show that $P$ is contiguous to a point $T$ of $I_{2}$.

Let $Q T$ denote an arc lying in $I$. The point set $Q T+P$ contains a simple closed curve or triune $J^{\prime}$ whose interior $I^{\prime}$ contains infinitely many points, no one contiguous to any point of $J$. Thus we reach a contradiction, and the theorem is established.

Example 1. In the euclidean plane let $C_{1}, C_{2}$, and $C_{3}$ denote three circles, each tangent externally to each of the others. Denote their centers by $P_{1}, P_{2}$, and $P_{3}$, respectively, and their radical center by $O$. Let $A_{1}=C_{1} \cdot C_{2}, A_{2}=C_{2} \cdot C_{3}$, and $A_{3}=C_{3} \cdot C_{1}$. Let $B_{1}, B_{2}$, and $B_{3}$ denote points on the rays $O A_{1}, O A_{2}$, and $O A_{3}$, respectively, such that $d\left(O, B_{1}\right)=d\left(O, B_{2}\right)=d\left(O, B_{3}\right)$, and $d\left(O, B_{1}\right)$ $>d\left(O, A_{1}\right)$. Let $B_{3} B_{1}, B_{1} B_{2}$, and $B_{2} B_{3}$ be circular arcs having $P_{1}, P_{2}$, and $P_{3}$, respectively, as centers and lying on the non- $O$ sides of the lines $B_{3} B_{1}, B_{1} B_{2}$, and $B_{2} B_{3}$, respectively. Let $\beta_{11}=B_{3} B_{1}+O B_{3}+O B_{1}, \beta_{21}=B_{1} B_{2}+O B_{1}+O B_{2}$, $\beta_{31}=B_{2} B_{3}+O B_{2}+O B_{3}$. For each point $P$ of $\beta_{i 1}$ and each $j, \quad(i=1,2,3$; $j=1,2,3, \cdots)$, let $Q_{P i j}$ denote the point of the interval $P_{i} P$ such that $d\left(P_{i}, Q_{P_{i j}}\right)=d\left(P_{i}, A_{i}\right)+\left[d\left(P_{i}, P\right)-d\left(P_{i}, A_{i}\right)\right] / j$, and let $\beta_{i j}$ denote the set of all points $Q_{P i j}$. Let $T_{i},(i=1,2,3)$, denote $C_{i}$ plus its interior. Let $S$ denote the following collection: (1) $T_{i},(i=1,2,3)$, is an element of $S$, and (2) each point of the plane not in $T_{1}+T_{2}+T_{3}$ is an element of $S$. For each positive integer $n$, let $G_{n}$ denote the following subsets of $S$ : (1) the interior of each circle of radius $1 / n$ or less which neither encloses nor contains a point of $T_{1}+T_{2}+T_{3}$ is an element of $G_{n}$; (2) for each pair $(i, j),(i=1,2,3$; $j=n, n+1, \cdots)$, the set consisting of $T_{i}$ and all elements of $S$ enclosed by $\beta_{i j}$ is an element of $G_{n}$. If each element of $S$ is called a "point," each element of $G_{1}$ is called a "region," and each of the "points" $T_{1}, T_{2}$, and $T_{3}$ is "contiguous to" each of the others, then each of the axioms of this paper is nonvacuously satisfied.

Example 2. In a euclidean space let $s_{1}, s_{2}, s_{3}, s_{4}$, and $s_{5}$ denote the spheres whose equations are $(x-1)^{2}+y^{2}+z^{2}=3 / 2^{2},(x+1 / 2)^{2}+\left(y-(3)^{1 / 2} / 2\right)^{2}+z^{2}=3 / 2^{2}$, and $(x+1 / 2)^{2}+\left(y+(3)^{1 / 2} / 2\right)^{2}+z^{2}=3 / 2^{2}, x^{2}+y^{2}+\left(z-(3)^{1 / 2} / 12\right)^{2}=3 / 12^{2}$, and $x^{2}+y^{2}+\left(z+(3)^{1 / 2} / 12\right)^{2}=3 / 12^{2}$, respectively. Each of these spheres is tangent externally to each of the others. For each $i,(i=1, \cdots, 5)$, let $T_{i}$ denote $s_{i}$ 
plus its interior. Let $S$ denote the collection defined as follows: (1) For each $i$, $(i=1, \cdots, 5), T_{i}$ is an element of $S$. (2) Let $K$ denote the $(x, y)$-plane. Each point of the unbounded component of $K-K\left(T_{1}+T_{2}+T_{3}\right)$ is an element of $S$. (3) For each set of three distinct positive integers $i, j$, and $k$, such that each integer is less than 6 and at least one integer is either 4 or 5 , let $M_{i j k}$ denote the plane which contains the centers of the spheres $s_{i}, s_{j}$, and $s_{k}$. Each point of the bounded component of $M_{i j k}-M_{i j k}\left(T_{i}+T_{j}+T_{k}\right)$ is an element of $S$. For each positive integer $n$, let $G_{n}$ denote the subsets of $S$ defined as follows: (1) The interior of each circle of radius $1 / n$ or less which is a subset of $S \cdot M_{i j k}$ or of $S \cdot K$ is an element of $G_{n}$. (2) In each of the sets $S \cdot M_{i j k}$, construct three sequences of segments like the three sequences of segments constructed within the triune of Example 1, and in $S \cdot K$ construct three sequences of segments like those constructed in the exterior of the triune of Example 1. For each pair $(i, j),(i=1, \cdots, 5 ; j=n, n+1, \cdots)$, the subset of $S$ consisting of $T_{i}$ together with all elements of $S-\left(T_{1}, T_{2}, \cdots, T_{5}\right)$ each of which is enclosed by $T_{i}$ plus the $j$ th segment of one of the sequences constructed is an element of $G_{n}$. If each element of $S$ is called a "point," each element of $G_{1}$ is called a "region," and each of the "points" $T_{1}, \cdots, T_{5}$ is "contiguous to" each of the others, then all the axioms of this paper are non-vacuously satisfied.

Example 2 shows that Theorem 6 fails to hold if $J$ denotes a triune instead of a simple closed curve.

THEOREM 18. If $J$ is a simple closed curve, if $I$ is the interior of $J$, and if $H$ and $K$ are mutually exclusive compact continua lying in $J+I$, then no two points of $H$ separate two points of $K$ on $J$.

Proof. Suppose on the contrary that the points $A$ and $B$ of $H$ separate the points $C$ and $D$ of $K$ on $J$. It follows from Theorem 24 of S.C.P. that $K$ contains an irreducible continuum $T$ from $K \cdot A C B$ to $K \cdot A D B$. By Theorem 28 of S.C.P., $T-T \cdot J$ is a connected set having boundary points $C_{1}$ and $D_{1}$ in segments $A C B$ and $A D B$, respectively. It may be readily shown that the component of $S-(J+H)$ which contains $T-T \cdot J$ contains a segment $C_{2} Y D_{2}$, where $C_{2}$ and $D_{2}$ are points of segments $A C B$ and $A D B$, respectively, and neither point belongs to $H$. Thus the arc $C_{2} Y D_{2}$ contains no point of $H$ and lies, except for its end points, in $I$.

The above argument may be repeated to show that there exists an arc $A_{2} X B_{2}$ which contains no point of $C_{2} Y D_{2}$, where the segment $A_{2} X B_{2}$ lies in $I$, and $A_{2}$ and $B_{2}$ separate $C_{2}$ and $D_{2}$ on $J$. But this contradicts Theorem 4 .

THEOREM 19. The interior of a simple closed curve or triune is not a subset of any simple closed curve or triune. 
Proof. Let $I$ denote the interior of a simple closed curve or triune $J$. By Theorem $5, I$ is not a subset of a triune. Suppose $I$ is a subset of a simple closed curve $C$. By Theorem 17, there exists a point $P$ of $I$ which is not contiguous to any point of $J$. Now $P$ is either a limit point of $S-C$ or is contiguous to a point of $S-C$. If $P$ is a limit point of $S-C$, let $R$ be a connected domain containing $P$, but containing no point of $J$, and let $Q$ be a point of $R \cdot(S-C)$. If $P$ is not a limit point of $S-C$, let $Q$ be a point of $S-C$ which is contiguous to $P$. In either case, $Q$ belongs to $I \cdot(S-C)$.

THEOREM 20. If neither of the contiguous points $X$ and $Y$ separates the point $A$ from the point $B$, then their sum does not separate $A$ from $B$.

Proof. Suppose $S-(X+Y)=S_{A}+S_{B}$, where $S_{A}$ and $S_{B}$ are mutually separated sets containing $A$ and $B$, respectively. There exists an arc $\alpha$ from $A$ to $B$ which does not contain $Y$. The arc $\alpha$ contains $X$; hence $\alpha$ contains an $\operatorname{arc} A X$. Similarly, there exists an $\operatorname{arc} A Y$. The point set $A X+A Y-(X+Y)$ is connected and hence lies in $S_{A}$. The set $A X+A Y$ contains a simple closed curve or triune $J$ which contains $X$ and $Y$ and is a subset of $S_{A}+X+Y$. Let $Q$ be any point of $J-(X+Y)$, and let $I$ and $E$ denote the two complementary domains of $J$. Since $Q$ is a point of $S_{A}$ and is a boundary point of each of the connected sets $I$ and $E$, it follows that $I$ and $E$ are subsets of $S_{A}$. Hence $S$ is a subset of $S_{A}+X+Y$. Thus we reach a contradiction, and the theorem is established.

Aхгом 4. If $P$ and $Q$ are two distinct non-contiguous points, there exists a simple curve or triune which separates $P$ from $Q$.

With the help of Axiom 4, the Borel-Lebesgue theorem (Theorem 5 of S.C.P.); and Theorem 16, the next theorem may be established.

THEOREM 21. If $H$ and $K$ are two mutually separated compact continua, there exists a simple closed curve or triune which separates $H$ from $K$.

THEOREM 22. If $J$ is a simple closed curve or triune containing the contiguous points $A$ and $B, I$ is the interior of $J$, and $P$ is a point of $J+I$ which is not contiguous to $A$, then there exists a simple closed curve or triune $J^{*}$ satisfying the following conditions: (1) It is a subset of $J+I$. (2) Its intersection with $J$ is an arc containing $A$ and $B$. (3) Its exterior contains $P$.

Proof. Suppose the theorem is false. If may be readily shown that there exists a simple closed curve or triune which satisfies conditions (1) and (2). Furthermore it may be readily shown that if $P$ is any point of $J$ not contiguous to $A$, or if $P$ is a point of $I$ not contiguous to $A$ such that there is a simple closed curve or triune $J^{\prime}$ containing $P$ and satisfying conditions (1) and (2), 
then there exists a simple closed curve or triune satisfying conditions (1), (2), and (3). Hence our supposition implies that $P$ is a point of $I$, and every simple closed curve or triune, satisfying conditions (1) and (2), encloses $P$. Let $P X$ denote an arc from $P$ to some point $X$ of $J-(A+B)$ such that $P X-X$ is a subset of $I$. Let $S_{1}$ denote the set consisting of $P$ and all points $Y$ of $P X-X$ such that every simple closed curve or triune satisfying conditions (1) and (2) encloses the interval $P Y$ of $P X$, and let $S_{2}$ denote $P X-S_{1}$. By the Dedekindcut proposition (P.S.T., chap. 1, Theorem 64) there exists a point $Q$ which is either the last point of $S_{1}$ or the first point of $S_{2}$, in the order $P$ to $X$.

Suppose $Q$ is the first point of $S_{2}$. There exists a simple closed curve or triune $J^{\prime}$ satisfying the following conditions: If $Q=X$, then $J^{\prime}=J$; if $Q \neq X$, then $J^{\prime}$ contains $Q$, satisfies conditions (1) and (2), and encloses $P Q-Q\left(=S_{1}\right)$. There exists a third simple closed curve or triune $J_{1}$, which is a subset of $J^{\prime}$ plus its interior and is such that $J^{\prime} \cdot J_{1}$ is an arc containing $A$ and $B$ but not containing $Q$. Thus $Q$ is exterior to $J_{1}$; hence $P Q-Q$ either intersects $J_{1}$ or is exterior to $J_{1}$. Both these possibilities are ruled out since $J_{1}$ satisfies conditions (1) and (2) and hence encloses $P Q-Q$. Thus $Q$ is not the first point of $S_{2}$.

Suppose $Q$ is the last point of $S_{1}$. By means of an argument analogous to that used in the last paragraph, it may be shown that $Q$ is not contiguous to a point of $S_{2}$. Thus $Q$ is a limit point of $S_{2}$. Now $Q$ cannot be contiguous to both $A$ and $B$; for if such were the case, the triune $A B Q$ would satisfy conditions (1) and (2) and hence would enclose $P Q=S_{1}$ and, in particular, the point $Q$. Let $C$ denote a point of the pair $(A, B)$ which is not contiguous to $Q$, and let $C \omega$ be an arc from $C$ to $\omega$ lying in $J$ plus its exterior and containing no point of $J$ which is contiguous to $Q$. By Theorem 21, there exists a simple closed curve or triune $J^{\prime}$ which separates $Q$ from $C \omega$. Since $Q$ is a limit point of $S_{2}$, it follows that $I^{\prime}$, the interior of $J^{\prime}$, contains a segment $Q W$ of $S_{2}$. There exist a point $F$ of the segment $Q W$, and a simple closed curve or triune $J_{1}$ which satisfies conditions (1) and (2) and is such that $F$ is in $E_{1}$, the exterior of $J_{1}$. Let $I_{1}$ denote the interior of $J_{1}$. By Theorem 12, there exists a simple closed curve or triune $J_{2}$, which is a subset of $J_{1}+J^{\prime}$ and whose interior $I_{2}$ contains $Q$ and is a subset of $I_{1} \cdot I^{\prime}$. There exists a point $T$ of $J_{2} \cdot I^{\prime} \cdot J_{1}$. It may be readily shown that the point set $J_{1}-T+\left(J_{2}-T\right)$ contains a simple closed curve or triune $J^{*}$ which satisfies conditions (1) and (2). Furthermore, since $I_{2}+T+E_{1}$ is a connected point set containing $Q$ and $\omega$ but no point of $J^{*}, Q$ is exterior to $J^{*}$. Hence $Q$ is not a point of $S_{1}$.

Thus we have reached a contradiction and the theorem is established.

THEOREM 23. The interior of every triune is non-compact and so is the interior of every simple closed curve which contains two contiguous points. 
Proof. Let $J$ denote a simple closed curve or triune containing the contiguous points $A$ and $B$, and let $I$ denote the interior of $J$. Suppose that $I$ is compact. It follows that $J+I$ is compact, completely separable, and metric. Let $D_{1}, D_{2}, D_{3}, \cdots$ be a sequence of domains which properly covers $J+I$ and with respect to which $J+I$ is completely separable. Let $n_{1}$ denote the smallest integer (which exists in view of Theorems 17 and 22) such that there exists a simple closed curve or triune $J_{1}$ which is a subset of $J+I$, and such that $J \cdot J_{1}$ is an arc containing $A$ and $B$, and $D_{n_{1}}$ is exterior to $J_{1}$. Let $n_{1}, n_{2}, n_{3}, \cdots$ be an increasing sequence of positive integers satisfying the following conditions: For each integer $k>1, n_{k}$ is the smallest integer greater than $n_{k-1}$ such that there exists a simple closed curve or triune $J_{k}$ which is a subset of $J_{k-1}$ plus its interior, and such that $J_{k-1} \cdot J_{k}$ is an arc containing $A$ and $B$, and $D_{n k}$ is exterior to $J_{k}$. For each integer $r$, let $\alpha_{r}$ denote the point $J_{r}-(A+B)$ in case $J_{r}$ is a triune. Otherwise, let $\alpha_{r}$ denote an arc $P_{r} Q_{r}$ of $J_{r}-(A+B)$, where $Q_{r}$ is either contiguous to $B$ or else $d\left(Q_{r}, B\right)<1 / r$, and where $P_{r}$ is either contiguous to $A$ or else $d\left(P_{r}, A\right)<1 / r$. For each integer $n$ let $E_{n}$ denote the exterior of $J_{n}$. For each integer $r$ and each point $P$ of $\alpha_{r}$ there exists an integer $n_{P}$ such that $E_{n_{P}}$ contains $P$. By the Borel-Lebesgue theorem, there exists a finite collection of these domains covering $\alpha_{r}$. The domain of this finite collection with greatest subscript $E_{s}$, contains all other domains of the finite collection; therefore $E_{s}$ contains $\alpha_{r}$. Hence $\alpha_{r} \cdot J_{s}=0$, and consequently $\alpha_{r} \cdot \alpha_{s}=0$. Thus there exists an increasing sequence of integers $r_{1}, r_{2}, r_{3}, \cdots$ such that for each integer $k>0, \alpha_{r_{k}} \cdot \alpha_{r_{k+1}}=0$; hence $\alpha_{r_{1}}, \alpha_{r_{2}}, \cdots$ is a sequence of mutually exclusive continua. There exists a subsequence of this sequence which converges to a sequential limiting set $L$ containing $A$ and $B$. Hence by Theorem 33 of S.C.P., $L$ is a perfect continuum and is therefore uncountable. For each integer $n, L$ is a subset of $J_{n}$ plus its interior. Hence if $L$ contains a point $X$ other than $A$ or $B$, then $X$ is contiguous to $A$ or $B$. But the set of all such points $X$ is at most countable by Theorem 14 of S.C.P. Hence $L$ is countably infinite or finite. We have thus reached a contradiction, and the theorem is established.

Theorem 24.* If $H$ and $K$ are two mutually separated, closed and compact point sets containing the points $A$ and $B$, respectively, then there exists a simple closed curve or triune $J$ which separates $A$ from $B$ such that $J \cdot(H+K)=0$.

Proof. Let $h_{A}$ and $k_{B}$ denote the components of $H$ and $K$ containing $A$ and $B$, respectively. From Theorem 21 it follows that for each component $h$ of $H$ there exists a simple closed curve or triune $J_{h B}$ which separates $h$ from

* Cf. L. Zoretti, Sur les fonctions analytiques uniformes, Journal de Mathématiques Pures et Appliquées, vol. 1 (1905), pp. 9-11. 
$k_{B}$. Let $D_{h B}$ denote that complementary domain of $J_{h B}$ which contains $h$. The collection of all such domains $D_{h B}$ covers $H$; hence there exists a finite collection $T$ of such domains covering $H$. Let $T^{*}$ denote the point set which is the sum of all the elements of $T$. By Theorem 16 there exists a simple closed curve or triune $J_{A B}$ whose interior with respect to $B$ contains that component of $T^{*}$ which contains $A$. Furthermore, $\left(H+k_{B}\right) \cdot J_{A B}=0$. For each component $k$ of $K$ let $J_{A k}$ denote a simple closed curve or triune (which exists in view of the preceding argument) which separates $A$ from $k$ and is such that $(H+K) \cdot J_{A k}=0$. Let $D_{A k}$ denote the interior of $J_{A k}$ with respect to $A$. The collection of all such domains $D_{A k}$ covers $K$; hence there exists a finite collection $G$ of such domains covering $K$. Let $G^{*}$ denote the sum of the elements of $G$. By Theorem 16 there exists a simple closed curve or triune $J$ whose interior with respect to $A$ contains that component of $G^{*}$ which contains $B$. Furthermore, $(H+K) \cdot J=0$.

THEOREM 25. $\dagger$ If $H$ and $K$ are two mutually separated closed and compact point sets, and if neither $H$ nor $K$ separates the point $A$ from the point $B$, then $H+K$ does not separate $A$ from $B$.

Proof. Suppose $H+K$ separates $A$ from $B$.

Case 1. Suppose $H$ is connected. With the help of Theorem 24, it may be shown that there exists a domain $D$ which contains $K$ but no point of $H$ and is the sum of a finite number of components each of which is bounded by a simple closed curve or triune containing no point of $H+K$. Let $A X B$ denote an arc from $A$ to $B$ containing no point of $K$, and $A Y B$ an arc containing no point of $H$. Let $J_{1}, J_{2}, J_{3}, \cdots, J_{r}$ denote the boundaries of those components of $D$ which have points in common with $A Y B$. There are four possibilities: (1) $A$ and $B$ are both in $S-D$, (2) $A$ and $B$ are the same component of $D$, (3) $A$ and $B$ are in different components of $D$, (4) $A$ or $B$ is in $S-D$ while the other is in $D$. In each case it may be readily shown that $(A Y B-A Y B \cdot D)$ $+J_{1}+J_{2}+\cdots+J_{r}+A X B \cdot D$ contains an arc from $A$ to $B$.

Case 2. Suppose $H$ has only a finite number of components, $h_{1}, h_{2}, \cdots, h_{n}$. By Case $1, h_{1}+K$ does not separate $A$ from $B$. The sets $h_{2}$ and $h_{1}+K$ are mutually separated; hence by Case $1, h_{2}+\left(h_{1}+K\right)$ does not separate $A$ from $B$. Continuing this process we obtain the fact that $h_{n}+\left(h_{n-1}+\cdots+h_{1}+K\right)$ does not separate $A$ from $B$.

Case 3. Suppose $H$ has infinitely many components. Let $A X B$ denote an arc containing no point of $K$. By Axiom C, at most a finite number of components of $K$ contain points which are contiguous to points of $A X B$. Let

† Cf. P. Alexandroff, Sur les multiplicités cantoriennes et le thêorème de Phragmèn-Brouwer généralisé, Comptes Rendus de l'Académie des Sciences, Paris, vol. 183, pp. 722-724. 
$k_{1}, k_{2}, \cdots, k_{n}$ be the set of all such components of $K$. By Case 2 there exists an $\operatorname{arc} A Y B$ which contains no point of $H+k_{1}+\cdots+k_{n}$. If $A Y B \cdot K=0$, the theorem is established. Suppose $A Y B \cdot K \neq 0$. For each component $k$ of $K$ which intersects $A Y B$, the set $H+A X B+K$ is the sum of two mutually separated sets, one containing $A$ and the other containing $k$, by Theorem 27 of S.C.P. By Theorem 24 there exists a simple closed curve or triune $J_{k}$ which separates $A$ from $k$ and is such that $J_{k} \cdot(H+A X B+K)=0$. Let $I_{k}$ denote the interior of $J_{k}$ with respect to $A$. The collection of all such domains $I_{k}$ covers the closed point set $A Y B \cdot K$; hence there exists a finite subcollection $T$ doing so. Let $T^{*}$ denote the sum of the elements of $T$. By Theorem 16, for each component $t$ of $T^{*}$ there exists a simple closed curve or triune $C_{t}$ which is a subset of the boundary of $t$ and whose interior with respect to $A$ contains $t$. Let $C_{1}, C_{2}, \cdots, C_{s}$ be the set of all simple closed curves or triunes thus obtained. The point set $\left(A Y B-A Y B \cdot T^{*}\right)+C_{1}+C_{2}+\cdots+C_{s}$ contains an $\operatorname{arc}$ from $A$ to $B$ which contains no point of $H+K$.

THeOREм 26. If $J$ is a simple closed curve, $I$ is a complementary domain of $J, H$ and $K$ are two mutually separated subcontinua of $J, \alpha$ and $\beta$ are the two components of $J-(H+K)$, and $C$ is a simple closed curve which separates $H$ from $K$, then there exists an arc $A X B$ such that $A$ and $B$ are points of $\alpha$ and $\beta$, respectively, and segment $A X B$ is a subset of $I \cdot C$.

Proof. Suppose the theorem is false. Let $\omega$ be a point of $S-(J+I+C)$, which exists by Theorem 19. Let $D$ denote the interior of $C$. One of the sets $H$ and $K$, say $H$, is a subset of $D$. Obviously, $C$ contains at least one point of each of the sets $\alpha, \beta$, and $I$. Let $P, Q, Z$, and $W$ denote points of $\alpha \cdot C, \beta \cdot C$, $H$, and $K$, respectively. Let $\alpha^{\prime}$ and $\beta^{\prime}$ denote continua which are subsets of $\alpha$ and $\beta$, respectively, and which contain $\alpha \cdot C$ and $\beta \cdot C$, respectively. Now, $\alpha^{\prime}+\beta^{\prime}+I \cdot C$ is the sum of two mutually separated continua $M_{1}$ and $M_{2}$ containing $\alpha^{\prime}$ and $\beta^{\prime}$, respectively. Let $E$ denote the last point of the $\operatorname{arc} P Z Q$, in the order $P$ to $Q$, which is either a point of $M_{1}$ or contiguous to a point of $M_{1}$; and let $F$ denote the first point of $P Z Q$ which is either a point of $M_{2}$ or contiguous to a point of $M_{2}$. It may be readily seen with the help of Theorem 4 that if $E$ and $F$ are distinct, then $E$ precedes $F$ in the order $P$ to $Q$. Suppose first that $E$ and $F$ are distinct non-contiguous points. The interval $E F$ of $P Z Q$ contains at least one point of $H$. Furthermore, there exists an arc $O L$ where $L$ is a point of segment $E F$ and $O L-L$ is a subset of $I-I \cdot C$. By Theorem 12 there exists a simple closed curve $J^{\prime}$ which is a subset of $J+C$ and contains $E F$, and whose interior $I^{\prime}$ is a subset of $I \cdot D$ and contains $O L-L$. Now $J^{\prime}$ contains no point of $K$, for otherwise $H+I^{\prime}+K$ would be a connected set containing no point of $C$. Thus $J^{\prime}$ contains an $\operatorname{arc} E^{\prime} F^{\prime}$, where $E^{\prime}$ and $F^{\prime}$ 
are points of segments $L E W$ and $L F W$, respectively, and the segment $E^{\prime} F^{\prime}$ is a subset of $I \cdot C$. Thus segment $E^{\prime} F^{\prime}$ is a subset of $M_{1}$ and also a subset of $M_{2}$. From this contradiction, it follows that $E$ and $F$ are identical or contiguous. Also $(E+F) \cdot\left(M_{1}+M_{2}\right)=0$. Similarly, if $T$ denotes the last point of $P W Q$ which is either a point of $M_{1}$ or contiguous to a point of $M_{1}$, and if $V$ denotes the first point of $P W Q$ which is either a point of $M_{2}$ or contiguous to a point of $M_{2}$, then $T$ and $V$ are identical or contiguous. Let $C^{\prime}$ denote a simple curve which separates $M_{1}$ from $M_{2}$. Let $P^{\prime} Q^{\prime}$ denote an arc such that $P^{\prime}$ and $Q^{\prime}$ are points of $M_{1}$ and $M_{2}$, respectively, and segment $P^{\prime} Q^{\prime}$ is a subset of $J+I-(J+I) \cdot(C+E+F+T+V)$. Let $G$ denote a point of segment $P^{\prime} Q^{\prime}$ which belongs to $C^{\prime}$, and let $N Y$ denote an arc which is a subset of $C^{\prime}$ and contains $G$, where $N$ and $Y$ are points of $J$ and segment $N Y$ is a subset of $I$. Now $N$ and $Y$ do not lie in different components of $J-\left(\alpha^{\prime}+\beta^{\prime}\right)$, for otherwise, $H+K+N Y$ would be a connected set containing no point of $C$. Suppose both $N$ and $Y$ are points of that component of $J-\left(\alpha^{\prime}+\beta^{\prime}\right)$ which contains $H$. If either $N$ or $Y$ preceded $E$ in the order $P Z Q$, we would have two arcs (one a subset of $C \cdot M_{1}+E$, the other a subset of $C \cdot M_{2}+$ interval $Q^{\prime} G$ of arc $P^{\prime} Q^{\prime}+$ interval $N G$ of arc $N Y$ ) satisfying the hypothesis of Theorem 4 but not the conclusion of this theorem. Similarly, it may be shown that neither $N$ nor $Y$ follows $F$. Hence $N$ is identical with $Y$ or contiguous to $Y$. Again a contradiction is reached, and the theorem is established.

TheOREM 27. If $A, X, B$, and $Y$ are points of the simple closed curve $J$ in the order indicated, if $I$ is the interior of $J$, and if $H$ and $K$ are mutually separated closed and compact subsets of $J+I$ such that $H \cdot A X B=0$ and $K \cdot A Y B=0$, then there exists an arc $A B$ lying in $J+I-(H+K)$.

Proof. Two cases will be considered.

Case 1. Suppose there exists no component $T$ of $H+K$ such that $T+J-(A+B)$ is connected. Let $N_{1}$ and $N_{2}$ denote continua which are subsets of segments $A Y B$ and $A X B$, respectively, and which contain $H \cdot A Y B$ and $K \cdot A X B$, respectively; and let $\alpha$ and $\beta$ denote the components of $J-\left(N_{1}+N_{2}\right)$ which contain $A$ and $B$, respectively. By Theorem 27 of S.C.P., $N_{1}+N_{2}+H+K$ is the sum of two mutually separated closed sets $M_{1}$ and $M_{2}$ containing $N_{1}$ and $N_{2}$, respectively. Hence by Theorem 24 there exists a simple closed curve $C$ which separates $N_{1}$ from $N_{2}$ and contains no point of $M_{1}+M_{2}$. Thus $C$ contains no point of $H+K$. By Theorem 26 there exists an arc $A^{\prime} X^{\prime} B^{\prime}$, where $A^{\prime}$ and $B^{\prime}$ are points of $\alpha$ and $\beta$, respectively; and segment $A^{\prime} X^{\prime} B^{\prime}$ lies in $I \cdot C$. Now $A^{\prime} X^{\prime} B^{\prime}+\alpha+\beta$ contains an arc $A B$ lying in $J+I-(H+K)$.

Case 2. Suppose that for at least one component $T$ of $H+K, T+J$ 
$-(A+B)$ is connected. With the help of Axiom $\mathrm{C}$, it may be shown that there is only a finite number of such components. Either $H$ or $K$, say $H$, contains at least one such component. There exists a point $Y_{1}$ which is the first point of segment $A Y B$, in order $A$ to $B$, which is either a point of, or contiguous to a point of, such a component of $H$.

First suppose there exists at least one point $Q$ of $A Y_{1}-A$ having the property that there is a component $T_{Q}$ of $K$ such that $Q+T_{Q}+$ segment $A X B$ is connected. Let $Q_{1}, Q_{2}, \cdots, Q_{n}$ denote all such points in the order $A$ to $Y_{1}$. Obviously $Q_{i},(i=1,2, \cdots, n)$, is not a point of $H+K$. If the segment $A Q_{1}$ of $A Y_{1}$ contains no point of $H$, let $\alpha_{1}$ denote the interval $A Q_{1}$. If segment $A Q_{1}$ contains a point of $H$, it may be shown with the help of Theorem 18 and Case 1 of this theorem that there exists an arc $\alpha_{1}$, from $A$ to $Q_{1}$, lying in $J+I-(H+K)$. Similarly, if $n>1$, there exists an arc $\alpha_{i},(i=2,3, \cdots, n)$, from $Q_{i-1}$ to $Q_{i}$, lying in $J+I-(H+K)$. The set $\alpha_{1}+\alpha_{2}+\cdots+\alpha_{n}$ contains an $\operatorname{arc} \alpha$ from $A$ to $Q_{n}$. Now let $X_{2}$ denote the last point of segment $A X B$, in order $A$ to $B$, for which there exists a component $T_{2}$ of $H$, such that $Y_{1}+T_{2}+X_{2}$ is connected. If $Q_{n}=Y_{1}$, and if there exists a point $P$ of $A X B$ between $X_{2}$ and $B$ for which there is a component $T^{\prime}$ of $K$ such that $Y_{1}+T^{\prime}+P$ is connected, then, denoting $Q_{n}$ by $Q^{\prime}$, we have an $\operatorname{arc} A Q^{\prime}=\alpha$ having the following properties: (1) The arc $A Q^{\prime}$ lies in $J+I-(H+K),(2) Q^{\prime}$ is a point of segment $A Y B$ and does not precede $Y_{1},(3)$ there is at least one component $T$ of $H$ for which [ $T+\operatorname{segment} A X B+\operatorname{interval} A Q^{\prime}($ of $A Y B)-A$ ] is connected, but $T+Q^{\prime} B-Q^{\prime}$ is not connected, and (4) there exists no component $W$ of $H+K$ such that $W+A Y B-Q^{\prime}$ is connected. If $Q_{n}=Y_{1}$, and if there exists no such point $P$, let $X_{1}$ denote the first point of $A X B$, such that $Y_{1}+T_{2}+X_{1}$ is connected; or if $Q_{k} \neq Y_{1}$, let $X_{1}$ denote the first point of $A X B$ for which there exists a component $T$ of $H$ such that $Y_{1}+T+X_{1}$ is connected. In either case, $X_{1}$ is a point of segment $A X B$, and with the help of Theorem 18, Case 1, it may be shown that there exists an arc $\beta$ from $Q_{n}$ to $X_{1}$ lying in $J+I-(H+K)$. If $X_{1} \neq X_{2}$, then by means of an argument like that used in obtaining $\alpha$, it may be shown that there exists an $\operatorname{arc} \gamma$, from $X_{1}$ to $X_{2}$, lying in $J+I-(H+K)$. If $X_{1}=X_{2}$, let $\gamma$ denote $X_{1}$. If there exists no point $X^{\prime}$ of $A X B$ between $X_{2}$ and $B$ for which there is a component $T$ of $K$ such that segment $A Y B+T+X^{\prime}$ is connected, then the argument for obtaining $\alpha$ may be used here to obtain an arc $\delta$, from $X_{2}$ to $B$, lying in $J+I-(H+K)$. In this case, $\alpha+\beta+\gamma+\delta$ contains an arc from $A$ to $B$, and the theorem is established. If there exists a point $X^{\prime}$, as described, let $Q^{\prime}$ denote the first point of segment $A Y B$ for which exists a component $T$ of $K$, such that $Q^{\prime}+T+$ segment $X_{2} B$ is connected. It follows from Theorem 18 and Case 1 , that there exists an arc $\eta$, from $X_{2}$ to $Q^{\prime}$, lying in $J+I-(H+K)$. In this case, $\alpha+\beta+\gamma+\eta$ con- 
tains an $\operatorname{arc} A Q^{\prime}$ having the four properties listed above.

Now suppose there is no point $Q$ as described above. Let $X_{2}$ have the same meaning as above, and let $\gamma$ denote an arc from $A$ to $X_{2}$ lying in $J+I-(H+K)$. Again, if there exists no point $X^{\prime}$, as described above, let $\delta$ denote an arc from $X_{2}$ to $B$ lying in $J+I-(H+K)$. In this case $\gamma+\delta$ contains an arc from $A$ to $B$, and the theorem is established. If there exists a point $X^{\prime}$, as described above, let $Q^{\prime}$ and $\eta$ be defined as before. Now $\gamma+\eta$ contains an arc $A Q^{\prime}$ with properties listed above.

Thus, in any case either there exists an arc $A B$ lying in $J+I-(H+K)$, or else there exists an $\operatorname{arc} A Q^{\prime}$ having the properties listed above. In the latter case, if there exists no point $Y^{\prime}$ of $A Y B$, between $Q^{\prime}$ and $B$, for which there exists a component $T$ of $H$ such that $Y^{\prime}+T+$ segment $A X B$ is connected, then the argument for obtaining $\alpha$ may be used to obtain an $\operatorname{arc} Q^{\prime} B$ lying in $J+I-(H+K)$. In such a case, $A Q^{\prime}+Q^{\prime} B$ contains an arc from $A$ to $B$, and the theorem is established. If there exists a point $Y^{\prime}$, as described above, let $Y_{2}$ denote the first such point in the order $Q^{\prime}$ to $B$. Now the argument for obtaining $A Q^{\prime}$ may be repeated to obtain either an arc $Q^{\prime} B$ lying in $J+I-(H+K)$ (in which case $A Q^{\prime}+Q^{\prime} B$ contains an arc from $A$ to $B$, and the theorem is established), or an $\operatorname{arc} Q^{\prime} Q^{\prime \prime}$ of such nature that $A Q^{\prime}+Q^{\prime} Q^{\prime \prime}$ contains an arc $A Q^{\prime \prime}$ having the following properties: (1) $A Q^{\prime \prime}$ lies in $J+I-(H+K),(2) Q^{\prime \prime}$ is a point of segment $A Y B$ and does not precede $Y_{2}$, (3) there are at least two components of $H$ such that if $T$ denotes either of them, then [T+segment $A X B+$ interval $A Q^{\prime \prime}$ of $\left.A Y B-A\right]$ is connected, but $\left[T+Q^{\prime \prime} B-Q^{\prime \prime}\right]$ is not connected, and (4) there exists no component $W$ of $H+K$, such that $W+A Y B-Q^{\prime \prime}$ is connected. Since there is only a finite number of components of $H$ such that if $T$ denotes any one of them, $T+J-(A+B)$ is connected, the above process may be repeated until we have obtained either an arc $A B$ lying in $J+I-(H+K)$, or an arc $A Q^{(k)}$ lying in $J+I-(H+K)$, where $Q^{(k)}$ is a point of segment $A Y B$, and there is no point $Y^{\prime}$ between $Q^{(k)}$ and $B$ for which there is a component $T$ of $H$ such that $Y^{\prime}+T+$ segment $A X B$ is connected. In the latter case, the argument for obtaining $\alpha$ may be used to obtain an arc $Q^{(k)} B$ lying in $J+I-(H+K)$. The set $A Q^{(k)}+Q^{(k)} B$ contains an arc $A B$ lying in $J+I-(H+K)$; and the theorem is established.

THEOREM 28.* If the common part of the closed and compact point sets $H$ and $K$ is a continuum, and if neither $H$ nor $K$ separates the point $A$ from the point $B$, and if furthermore, $H-H \cdot K$ and $K-H \cdot K$ are mutually separated sets, then $H+K$ does not separate $A$ from $B$.

* Cf. S. Janiszewski, Sur les coupures du plan faites par des continus, Prace MatematycznoFizyczne, vol. 26, 1913. Also Anna M. Mullikin, Certain theorems relating to plane connected point sets, these Transactions, vol. 24 (1922), pp. 144-162. 
Proof. Suppose on the contrary that $H+K$ separates $A$ from $B$. Let $M=H+K$ and $T=H \cdot K$. Let $S_{1}$ and $S_{2}$ denote the components of $S-M$ which contain $A$ and $B$, respectively. There exist $\operatorname{arcs} A X B$ and $A Y B$ such that $A X B \cdot H=0$ and $A Y B \cdot K=0$. Let $X_{1}$ and $Y_{1}$ denote the first points of $A X B$ and $A Y B$, respectively, which belong to the boundary of $S_{2}$. Thus $X_{1}$ and $Y_{1}$ belong to $K-T$ and $H-T$, respectively, and hence are not contiguous to each other. Let $A X_{1}$ and $A Y_{1}$ denote intervals of $A X B$ and $A Y B$, respectively. The set $A X_{1}+A Y_{1}$ contains an $\operatorname{arc} X_{1} A_{1} Y_{1}$ such that $X_{1} A_{1} \cdot H=0$ and $A_{1} Y_{1} \cdot K=0$. If $X_{1}$ is contiguous to any point of $S_{2}$, let $X_{2}$ denote such a point, and let $X_{1} X_{2}$ denote the arc consisting of these two points. Otherwise, let $R$ denote a connected domain containing $X_{1}$ but containing no point or boundary point (other than $X_{1}$ ) of $H+A_{1} Y_{1}$, let $X_{2}$ denote a point of $R \cdot S_{2}$, and let $X_{1} X_{2}$ denote an arc lying in $R$. If $Y_{1}$ is contiguous to any point of $S_{2}$, let $Y_{2}$ denote such a point, and let $Y_{1} Y_{2}$ denote the arc consisting of these two points. Otherwise, let $W$ denote a connected domain containing $Y_{1}$ but containing no point or boundary point (other than $Y_{1}$ ) of $K+A_{1} X_{1}+X_{1} X_{2}$, let $Y_{2}$ denote a point of $W \cdot S_{2}$, and let $Y_{1} Y_{2}$ denote an arc lying in $W$. Let $X_{2} Y_{2}$ denote either the point $X_{2}$ or an arc lying in $S_{2}$ according as $X_{2}$ is or is not $Y_{2}$. It may be readily shown that the set $X_{1} A_{1} Y_{1}+X_{1} X_{2}+Y_{1} Y_{2}+X_{2} Y_{2}$ contains a simple closed curve $J$ such that (1) $J$ contains $A_{1}$ and a point $B_{1}$ of $X_{2} Y_{2}$, and (2) of the two segments of $J$ from $A_{1}$ to $B_{1}$, one contains no point of $H$ and the other contains no point of $K$. Let $I$ denote that complementary domain of $J$ which does not contain $T$, and let $H_{1}=H \cdot(J+I)$ and $K_{1}=K \cdot(J+I)$. It follows from Theorem 27 that there exists an $\operatorname{arc} A_{1} B_{1}$ lying in $J+I-\left(H_{1}+K_{1}\right)$. Thus $A_{1} B_{1}$ contains no point of $M$. But this is impossible since $A_{1}$ and $B_{1}$ lie in different complementary domains of $M$. The theorem is thus established.

THEOREM 29. If no point of the arc $X Y$ separates the point $A$ from the point $B$, then $X Y$ does not separate $A$ from $B$.

Proof. Suppose $S-X Y=S_{A}+S_{B}$, where $S_{A}$ and $S_{B}$ are mutually separated sets containing $A$ and $B$, respectively. Let $S_{1}$ denote the set consisting of $X$ together with all points $Z$, if there are any, such that the interval $X Z$ does not separate $A$ from $B$, and let $S_{2}=X Y-S_{1}$. Now $S_{2}$ contains $Y$, and clearly every point of $S_{1}$ precedes every point of $S_{2}$. Hence there exists either a last point of $S_{1}$ or a first point of $S_{2}$.

Suppose there exist a point $O$ which is the last point of $S_{1}$ and $Q$ which is the first point of $S_{2}$. By. Theorem 20 the interval $O Q$ does not separate $A$ from $B$. Hence by Theorem 28, $S_{1}+O Q$ does not separate $A$ from $B$. Thus $X Q$ does not separate $A$ from $B$, contrary to the fact that $Q$ is a point of $S_{2}$. 
Suppose that there exists a point $O$ which is the last point of $S_{1}$. Let $O_{1}, O_{2}, O_{3}, \cdots$ be a sequence of points of $S_{2}$ converging to $O$ such that each precedes the next in the order $Y$ to $X$. By Theorem 28, the interval $O O_{n}$ separates $A$ from $B$. Thus, by Theorem 5 , Chapter 2, of P.S.T., the point $O$ separates $A$ from $B$.

Suppose there exists a point $O$ which is the first point of $S_{2}$. Let $O_{1}, O_{2}, O_{3}, \cdots$ be a sequence of points of $S_{1}$ converging to $O$ such that each precedes the next in the order $X$ to $Y$. By Theorem 28, the interval $O_{n} O$ separates $A$ from $B$. Hence by Theorem 5, Chapter 2, of P.S.T., the point $O$ separates $A$ from $B$. Again a contradiction is reached and the theorem is established.

The following proposition is false:

Proposition. If $A, B$, and $C$ are three distinct points, and if $A$ is not contiguous to either $B$ or $C$, then there exists a simple closed curve or triune which separates $A$ from $B+C$.

In Example 1, let $A$ be any point of the triune $T_{1} T_{2} T_{3}$, and let $B$ and $C$ be points of different complementary domains of this triune. There does not exist a simple closed curve or triune which separates $A$ from $B+C$.

Agricultural and Mechanical College of Texas, College Station, Texas 